\title{
A Simulation Tool for Dynamic Contrast Enhanced MRI
}

\author{
Nicolas Adrien Pannetier ${ }^{1,2 * 9}$, Clément Stéphan Debacker ${ }^{1,2 \uparrow}$, Franck Mauconduit ${ }^{1,2}$, Thomas Christen ${ }^{3}$, \\ Emmanuel Luc Barbier ${ }^{1,2}$
}

1 Institut National de la Santé et de la Recherche Médicale, U836, Grenoble, France, 2 Université Joseph Fourier, Grenoble Institut des Neurosciences, Grenoble, France, 3 Stanford University, Department of Radiology, Stanford, California, United States of America

\begin{abstract}
The quantification of bolus-tracking MRI techniques remains challenging. The acquisition usually relies on one contrast and the analysis on a simplified model of the various phenomena that arise within a voxel, leading to inaccurate perfusion estimates. To evaluate how simplifications in the interstitial model impact perfusion estimates, we propose a numerical tool to simulate the MR signal provided by a dynamic contrast enhanced (DCE) MRI experiment. Our model encompasses the intrinsic $R_{1}$ and $R_{2}$ relaxations, the magnetic field perturbations induced by susceptibility interfaces (vessels and cells), the diffusion of the water protons, the blood flow, the permeability of the vessel wall to the the contrast agent (CA) and the constrained diffusion of the CA within the voxel. The blood compartment is modeled as a uniform compartment. The different blocks of the simulation are validated and compared to classical models. The impact of the CA diffusivity on the permeability and blood volume estimates is evaluated. Simulations demonstrate that the CA diffusivity slightly impacts the permeability estimates ( $<5 \%$ for classical blood flow and CA diffusion). The effect of long echo times is investigated. Simulations show that DCE-MRI performed with an echo time $T_{E}=5 \mathrm{~ms}$ may already lead to significant underestimation of the blood volume (up to $30 \%$ lower for brain tumor permeability values). The potential and the versatility of the proposed implementation are evaluated by running the simulation with realistic vascular geometry obtained from two photons microscopy and with impermeable cells in the extravascular environment. In conclusion, the proposed simulation tool describes DCE-MRI experiments and may be used to evaluate and optimize acquisition and processing strategies.
\end{abstract}

Citation: Pannetier NA, Debacker CS, Mauconduit F, Christen T, Barbier EL (2013) A Simulation Tool for Dynamic Contrast Enhanced MRI. PLoS ONE 8(3): e57636. doi:10.1371/journal.pone.0057636

Editor: Angelique Louie, University of California Davis, United States of America

Received September 20, 2012; Accepted January 23, 2013; Published March 14, 2013

Copyright: (c) 2013 Pannetier et al. This is an open-access article distributed under the terms of the Creative Commons Attribution License, which permits unrestricted use, distribution, and reproduction in any medium, provided the original author and source are credited.

Funding: This work was funded by the Institut National du Cancer, the Association pour la Recherche sur le Cancer and the Ministry for Higher Education and Research of France. The funders had no role in study design, data collection and analysis, decision to publish, or preparation of the manuscript.

Competing Interests: The authors have declared that no competing interests exist.

*E-mail: nicolas.pannetier@gmail.com

9 These authors contributed equally to this work.

- These authors are joint senior authors on this work.

\section{Introduction}

Bolus-tracking MRI techniques are widely used in clinical and preclinical studies to obtain imaging biomarkers that predict tumors progression and outcome [1,2]. Depending on the predominant contrast in use, two different techniques can be employed: $T_{1}$-weighted dynamic contrast enhanced MRI (DCEMRI) [3] or $T_{2}^{*}$-weighted dynamic contrast susceptibility (DSCMRI) [4]. DSC-MRI is the approach of choice for measuring perfusion biomarker in the brain. DCE-MRI is preferred in other organs $[5,6]$ where the contrast agent (CA) leaks outside of the vessels. It is also used to assess the vessel permeability in the brain when the blood brain barrier (BBB) is disrupted. These techniques emerged at the same time about 20 years ago but their quantification remains challenging.

In a brain voxel with intact $\mathrm{BBB}$, the $\mathrm{CA}$ yields a transient, strong increase in voxel $R_{2}$ and $R_{2}^{*}$ due to the increase in the magnetic susceptibility difference $(\Delta \chi)$ between blood and tissue, and a more subtle increase in voxel $R_{1}$ due to the increase in blood $R_{1}$ and the water exchange between intra and extravascular compartments [7]. In a tissue with an altered BBB, the CA leaks across the vessels and $\Delta \chi$ is reduced at the vessel wall, limiting the increase in voxel $R_{2}^{*}$ whereas the $R_{1}$ effect is enhanced.
Additionally, the distribution of CA around extravascular impermeable cells further perturbs the magnetic field and increases $R_{2}^{*}$ which then competes with the $R_{1}$ enhancement. The intricacy of these phenomena makes the MR signal interpretation arduous.

In DCE-MRI, the analysis is made with compartment models which handle blood flow and CA exchanges but often lack methods to deal with the NMR signal. Ideally, one would combine the compartment models which describe the microscopic $R_{1}$ and $R_{2}$ changes [8-11] with a model that describes the perturbations of the magnetic field induced by the susceptibility interfaces [1215]. Recent progress to untangle these phenomena have been made by measuring DSC-MRI and DCE-MRI simultaneously using multi-echo sequences [16,17]. The analysis of these acquisitions requires the use of advanced analytical models [18] that can potentially provide new biomarkers [19]. However, the impact of the CA diffusion within the extravascular space on the MR signal is disregarded and the effect of the arising susceptibility gradients around the cells remains unclear. A better description of the entanglement of these various effects within a voxel is thus of considerable interest.

In this article we report a numerical model of the MR signal in a DCE-MRI like experiment acquired with a multi gradient-echo sequence over several minutes. Within an affordable computing 
time, the proposed approach considered: the effect of the magnetic field perturbations caused by vessel and cell interfaces, the diffusion of water molecules, the blood flow, the CA leakage across the vessel wall and the CA diffusion within the extravascular space. The CA distribution is considered uniform within the vascular compartment (plug-flow or not well mixed compartment are not considered). The algorithm relies on:

1. A compartment model to simulate the CA exchange between the capillary bed and the tissue.

2. The computation of the magnetic field induced by the susceptibility variations using a Fourier based approach [20,21].

3. The deterministic approach introduced by Bandetini et al. [22] and further developed by Klassen et al [23] to model the MR signal provided by free diffusing water molecules within an inhomogeneous magnetic field.

The impermeable vascular and cellular membranes were handled by a modified convolution kernel approach. The model was first validated and compared to simple cases where analytical solutions exist. The impact of the CA diffusion on the permeability estimate was then investigated and the flexibility of the tool was eventually demonstrated by running the simulation on vascular networks obtained from optical microscopy and on geometries with extravascular cells.

\section{Methods}

\section{Algorithm}

Simulations were performed in the Matlab environment (Mathworks Inc. Natick, MA, USA) on a Dell Precision computer (double quad $2.33 \mathrm{GHz}$ Intel Xeon processor, RAM $32 \mathrm{~GB}$ ) and at the CNRS/IN2P3 Computing Center (Lyon/Villeurbanne France). The step time was set to $\delta t=0.5 \mathrm{~ms}$ and the total duration of the simulated DCE experiment was $T_{F}=900 \mathrm{~s}$. To simulate a 900s-long DCE experiment $\left(1.8 .10^{6}\right.$ frames given our $\left.d t\right)$ with moderate computation time, simulations were performed in 2D. The overall time was about 14 days for a single DCE experiment. In the following, bold capital letters refer to 2D-lattices. II refers to the lattice filled with $1, \times$ to the point wise multiplication, $\otimes$ to the convolution and $\sum$ to the $2 \mathrm{D}$ summation over a lattice. Summation of a lattice and a scalar is done point wise.

The simulation tool was organized in three distinct blocks: Geometry, Physiology and NMR (Fig. 1, Table 1).

(a) Geometry block. The geometry was designed on a 2D plane sampled with $Q^{2}$ pixels. $N$ vessels with radius $R$ were randomly spread out orthogonal to the plane under periodic boundary condition, defining the blood surface fraction $B V f$ (equivalent to a volume fraction in 3D).

Cells were spread out with the same boundary condition and occupy a surface defined by the porosity $\Pi=1-($ Cellsurface + Vesselsurface $) /($ Planesurface $)$. The cell radius was initially set to $10 \mu \mathrm{m}$ and slowly shrunk to obtain the desired porosity.

$\mathbf{G}_{\mathbf{v}}$ denoted the lattice with 1 inside the vessels and 0 outside. $\mathbf{G}_{\mathbf{c}}$ denoted the lattice with 1 inside the cells and 0 outside.

(b) Physiology block. This block modeled the CA concentration, $\mathbf{C}(t)$, within the plane.

(b.1) Plasma. The time evolution of the concentration of CA in the vessels, $c_{v}(t)$, was described by the discretized form of a two compartments model where the CA enters the tissue via the arterial influx, leaves by venous outflux and exchange by transendothelial leakage. No spatial variation of CA caused by plug-flow or not well-mixed space are considered.

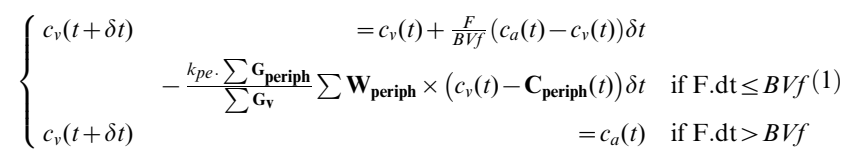

where $F$ denotes the blood flow in surface fraction per second (equivalent to a volume fraction in $3 \mathrm{D}), c_{a}(t)$ the arterial input function (AIF) and the terms modeling for the permeability are defined in the following paragraph. $c_{v}(t)$ was considered the same in each vessel. The AIF was an input to the physiology block.

(b.2) Permeability. The CA exchanges between the vessels and the extravascular space occurred only at the periphery of each vessel. $\mathbf{G}_{\text {periph }}$ was defined as the lattice with 1 in the one-pixel wide periphery of each vessel (connectivity 4 ) and with 0 outside. The CA concentration lattice in that region was denoted $\mathbf{C}_{\text {periph }}(t)$. At each time step, we considered the CA exchange between the vessels and its periphery. The amount of CA that extravasates was modeled by a first order kinetic law with exchange rate $\mathbf{k}_{\mathbf{p e}}$. The same exchange rate was considered in both ways:

$$
\mathbf{C}_{\text {periph }}(t+\delta t)=\mathbf{k}_{\text {pe }} \times\left(c_{v}(t)-\mathbf{C}_{\text {periph }}(t)\right) \delta t+\mathbf{C}_{\text {periph }}(t)
$$

In $3 \mathrm{D}$, one generally defines $k_{p e}$ as the exchange rate between the vessel and the extravascular extracellular volume, $v_{e}\left(k_{p e}=P . S / v_{e}\right.$, with $P$ the permeability and $S$ the surface exchange). In our $2 \mathrm{D}$ approach, we must consider the volume in which the CA extravasates, which is reduced to the surface $\sum \mathbf{G}_{\text {periph }}$, plus the contact exchange which is not equivalent for every points in the periphery of the vessels. Thereby, to remain consistent with the literature, $k_{p e}$ was scaled by $\mathbf{W}_{\text {periph }}$ :

$$
\mathbf{k}_{\text {pe }}=k_{p e} \mathbf{W}_{\text {periph }}
$$

with

$$
\mathbf{W}_{\text {periph }}=\frac{\sum\left(\mathbf{I I}-\mathbf{G}_{\mathbf{v}}-\mathbf{G}_{\mathbf{c}}\right)}{\sum \mathbf{G}_{\text {periph }}} \times \frac{\mathbf{S}}{\sum \mathbf{S}}
$$

where the first fraction accounts for the volume scaling and the second for the differences in the contact exchange. $\mathbf{S}$ was computed as (see also Fig. 2C for an illustration of $\mathbf{S}$ ):

$$
\mathbf{S}=\mathbf{G}_{\mathbf{v}} \otimes \mathbf{W}_{+}-\mathbf{G}_{\mathbf{v}} \quad \text { with } \quad \mathbf{W}_{+}=\left(\begin{array}{ccc}
0 & 1 & 0 \\
1 & 1 & 1 \\
0 & 1 & 0
\end{array}\right)
$$

The concentration lattice $\mathbf{C}$ was eventually updated with $\mathbf{C}_{\text {periph }}$ :

$$
\mathbf{C}=\mathbf{C} \times\left(\mathbf{I I}-\mathbf{G}_{\text {periph }}\right)+\mathbf{C}_{\text {periph }} \times \mathbf{G}_{\text {periph }}
$$

(b.3) CA Diffusion. The diffusion of CA into the extravascular space was modeled with a Gaussian diffusion kernel, denoted $\mathbf{D}_{\mathbf{C A}}$, as described by Eq.[7]:

$$
\mathbf{C}(t+\delta t)=\mathbf{C}(t) \otimes \mathbf{D}_{\mathbf{C A}} \quad \text { with } \quad \mathbf{D}_{\mathbf{C A}} \propto \frac{1}{\sigma^{2}} e^{-\frac{x^{2}+y^{2}}{2 \sigma^{2}}}
$$




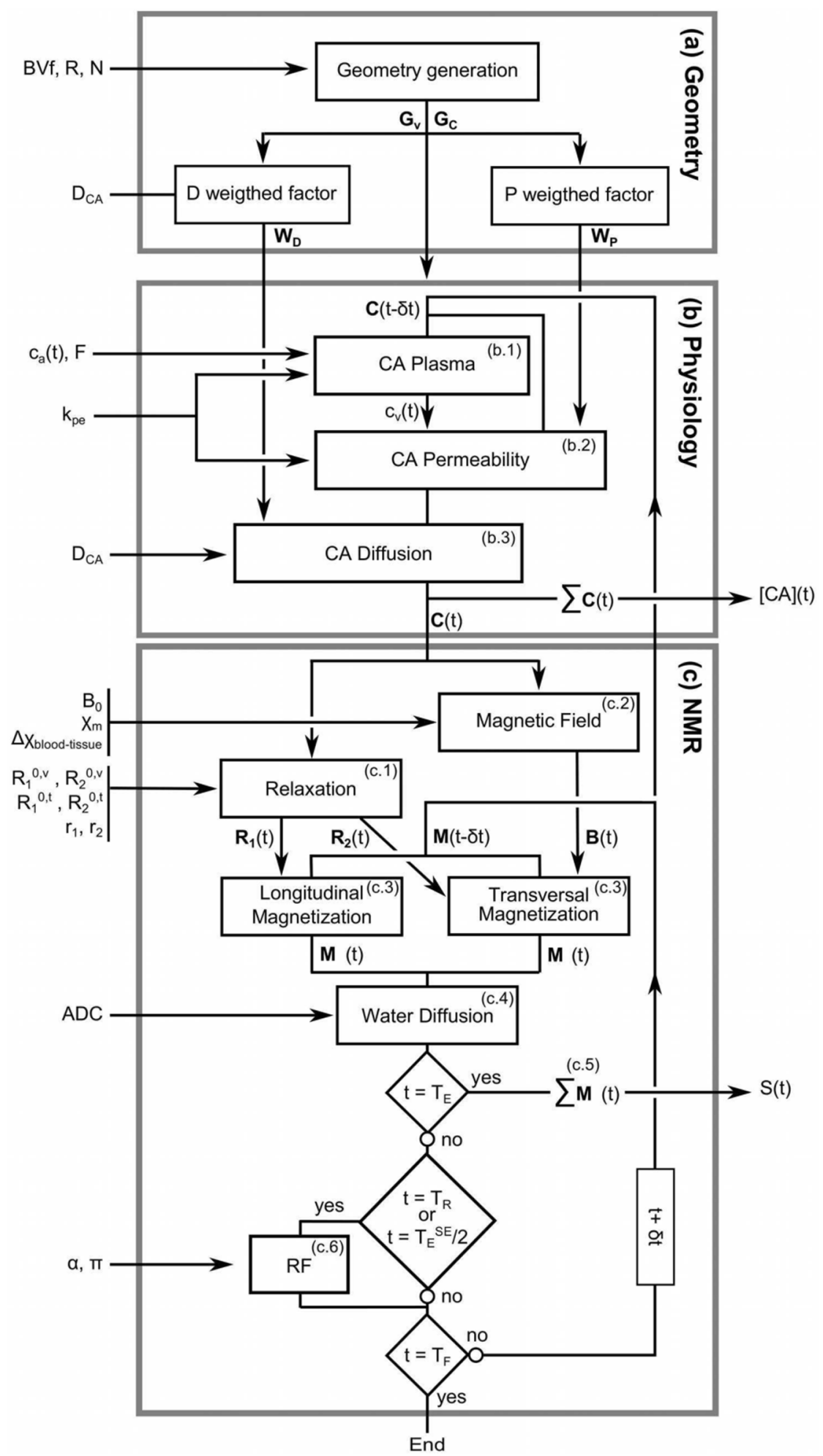

Figure 1. Algorithm sketch of the simulation. Only the most important parameters have been represented. Data on the left of the gray boxes are inputs to the model. Data on the right are outputs of the simulation. The simulation is organized in three blocks. Block (a) initializes the geometry. Block (b) describes the CA behavior over time. Block (c) estimates the MR signal. doi:10.1371/journal.pone.0057636.g001

where the mean square displacement of a CA molecule is $\left\langle x^{2}+y^{2}\right\rangle=2 \sigma^{2}=4 D_{C A} \delta t$ (in 2D, [22]) and $(x, y)$ the coordinates in the plane. The kernel $\mathbf{D}_{\mathbf{C A}}$ was designed with the same size as C. 
Table 1. List of the main parameters used in the algorithm.

\begin{tabular}{|c|c|c|}
\hline Name & Definition & Units \\
\hline$N$ & Number of vessels & - \\
\hline$B V f$ & Blood Volume fraction & $\%$ \\
\hline$R$ & Radius of the vessel & $\mu m$ \\
\hline$D_{C A}$ & Diffusivity of the CA & $\mu m^{2} \cdot s^{-1}$ \\
\hline $\mathbf{G}_{\mathrm{v}}$ & Vessel lattice & - \\
\hline $\mathbf{G}_{\mathbf{c}}$ & Cells lattice & - \\
\hline $\mathbf{S}$ & Contact surface lattice & - \\
\hline $\mathbf{W}_{\text {periph }}$ & Permeability weighting lattice & - \\
\hline $\mathbf{W}_{\mathrm{CA}}$ & Diffusion weighting lattice & - \\
\hline$c_{a}(t)$ & Arterial input function & $m M \cdot s^{-1}$ \\
\hline$F$ & Blood flow & $\% . s^{-1}$ \\
\hline$k_{p e}$ & Permeability rate & $s^{-1}$ \\
\hline$c_{v}(t)$ & Plasma CA concentration & $m M$ \\
\hline $\mathbf{C}(t)$ & CA concentration lattice & $m M$ \\
\hline$[C A](t)$ & Mean CA concentration in $\mathbf{C}(t)$ & $m M$ \\
\hline$B_{0}$ & Static magnetic field & $T$ \\
\hline$A D C$ & Apparent Diffusivity of the water & $\mu m^{2} \cdot s^{-1}$ \\
\hline$\alpha$ & RF flip angle & $\mathrm{rad}$ \\
\hline$\phi$ & RF phase angle & rad \\
\hline$\chi_{m}$ & Molar magnetic susceptibility of the $C A$ & ppm.m. $M^{-1}$ \\
\hline$\Delta \chi_{\text {blood-tissue }}$ & $\begin{array}{l}\text { Original Magnetic susceptibility between blood and } \\
\text { tissue }\end{array}$ & ppm \\
\hline$S(t)$ & MR signal & - \\
\hline $\mathbf{R}_{\mathbf{i}}(t)$ & Relaxation rates lattices & $s^{-1}$ \\
\hline $\mathbf{B}(t)$ & Magnetic field lattice & $T$ \\
\hline $\mathbf{M}_{\mathbf{i}}(t)$ & Magnetization lattices & - \\
\hline
\end{tabular}

The diffusion of CA described by Eq.[7] should neither contribute to the transendothelial transport modeled by Eq.[2] nor diffuse within the cells. We thus introduced a bounce-like mechanism for CA transport at the membranes of the vessels and cells characterized by the following weighting lattice (see also illustration in Fig. 2A):

$$
\mathbf{W}_{\mathbf{C A}}=\left(\left(\mathbf{G}_{\mathbf{v}}+\mathbf{G}_{\mathbf{c}}\right) \otimes \mathbf{D}_{\mathbf{C A}}\right) \times\left(\mathbf{I I}-\mathbf{G}_{\mathbf{v}}-\mathbf{G}_{\mathbf{c}}\right)
$$

For each point of the lattice, $\mathbf{W}_{\mathbf{C A}}$ defines the amount of $\mathrm{CA}$ that would have diffused from one point into the cells or vessels in the case of free diffusion. At each time step, this amount has to be sent back to the extravascular extracellular space. In our case, it is sent back to where it originates. The evolution of $\mathbf{C}(t)$ was thus

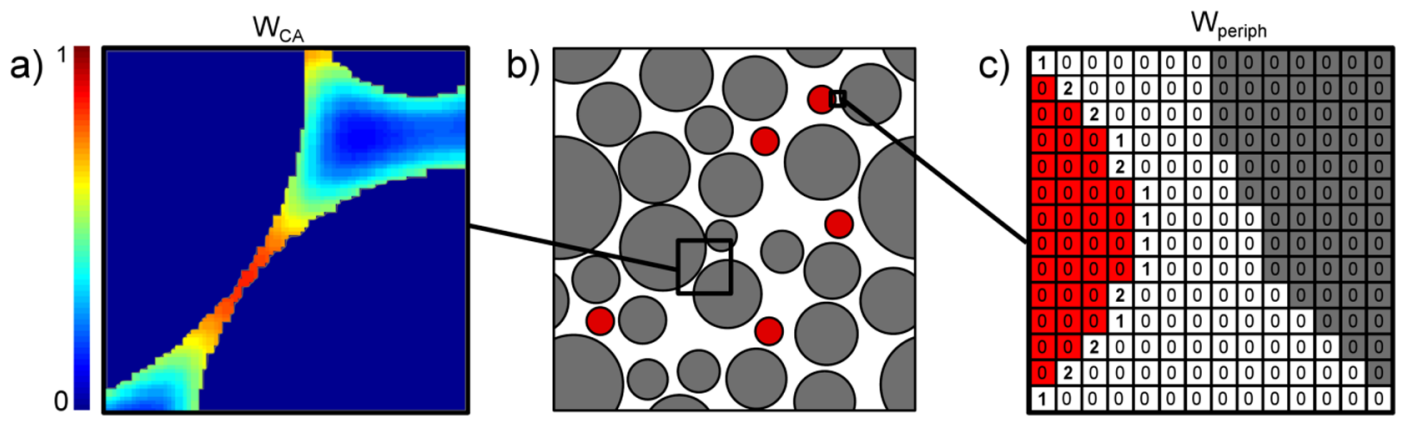

Figure 2. Illustration of the weighting lattices $\mathbf{S}$ and $\mathbf{W}_{\mathbf{C A}}$. (a) Zoom in the diffusion weighting lattice $\mathbf{W}_{\mathbf{C A}}$. The diffusion appears restricted near the membranes. (b) Illustration of the geometry lattices. In red, the vessel, in grey the cells. (c) Zoom in the surface weighting lattice $\mathbf{S}$ that computes the number of contact exchange interfaces between a vessel and its periphery.

doi:10.1371/journal.pone.0057636.g002 
computed with Eq.[9]:

$$
\begin{aligned}
& \mathrm{C}(t+\delta t)=\left(\mathrm{C}(t) \otimes \mathrm{D}_{\mathrm{CA}}+\mathrm{C}(t) \times \mathrm{W}_{\mathrm{CA}}\right) \times \\
& \left(\mathbf{I I}-\mathrm{G}_{\mathrm{v}}-\mathrm{G}_{\mathrm{c}}\right)+\mathbf{c}_{\mathbf{v}}(\mathbf{t}) \times \mathrm{G}_{\mathrm{v}}
\end{aligned}
$$

Special attention was paid to the kernel width to minimize physically impossible behaviors of CA such as jump over obstacles. The step time $\delta t$ was set according to $\sqrt{2 D_{C A} \delta t}<s_{m}$ where $s_{m}$ was the characteristic size of the smallest obstacles (see discussion section for further details). Each step that involved a convolution was computed using the FFT algorithm. The mean CA concentration in the plane, $[C A](t)$, was computed by averaging $\mathbf{C}(t)$.

(c) NMR block. This block modeled the magnetiztion, $\mathbf{M}(t)$, within the plane.

(c.1) Relaxation. Longitudinal and transverse relaxation lattices were calculated from $\mathbf{C}(t)$ based on Eq.[10]:

$$
\mathbf{R}_{\mathbf{i}}(t)=R_{i}^{0, v} \times \mathbf{G}_{\mathbf{v}}+R_{i}^{0, t} \times\left(\rrbracket-\mathbf{G}_{\mathbf{v}}\right)+r_{i} \mathbf{C}(t)
$$

where index $\mathrm{i}$ stands for 1 or $2, R_{i}^{0, v}$ and $R_{i}^{0, t}$ are the initial relaxation rates of the vascular and tissue compartments respectively and $r_{i}$ is the CA relaxivity.

(c.2) Magnetic field. The perturbations of the magnetic field induced by the susceptibility variations in the plane were computed using a Fourier based approach [20,21] adapted here in $2 \mathrm{D}, \mathrm{Eq} \cdot[11]$ :

$$
\Delta \tilde{\mathbf{B}}(t)=B_{0}\left(\frac{1}{3}-\frac{k_{y}^{2} \sin ^{2} \theta}{k_{x}^{2}+k_{y}^{2}}\right) \Delta \tilde{\chi}(t)
$$

where $k_{x}$ and $k_{y}$ are the coordinates in the Fourier space, $\theta$ the angle between the normal to the plane and $B_{0}$ and the tilde, $\tilde{X}$, denotes the Fourier transform of $\mathrm{X}$. The susceptibility map was defined by $\boldsymbol{\Delta} \chi(t)=\chi_{m} \mathbf{C}(t)+\Delta \chi_{\text {blood-tissue }} \times \mathbf{G}_{\mathbf{v}}$ where $\chi_{m}$ is the molar magnetic susceptibility of the $\mathrm{CA}$ and $\Delta \chi_{\text {blood-tissue }}$ the original magnetic susceptibility difference between the vessel and tissue. The perturbation of the magnetic field was averaged over 3 orthogonal orientations of the plane with respect to $B_{0}$ to mimic an isotropic distribution of vessels in a $3 \mathrm{D}$ voxel (see validation in results section).

(c.3) Magnetization. The magnetization evolution was described by the Bloch equations, Eq.[12]:

$$
\left\{\begin{array}{r}
\mathbf{M}_{\perp}(t+\delta t) \quad=\mathbf{M}_{\perp}(t) e^{-i \gamma \mathbf{B}(t) \delta t} e^{-\mathbf{R}_{2}(t) \delta t} \\
\mathbf{M}_{\|}(t+\delta t) \quad=\left(\mathbf{M}_{\|}(t)-\mathbf{M}_{0}\right) e^{-\mathbf{R}_{1}(t) \delta t}+\mathbf{M}_{0}
\end{array}\right.
$$

with $\gamma=2.68 \cdot 10^{8} \mathrm{rad} \cdot \mathrm{s}^{-1} \cdot T^{-1}$ and $\mathbf{M}_{0}$ the longitudinal magnetization at equilibrium. The symbol $e$ denotes here a point wise exponentiation.

(c.4) Water diffusion. The diffusion of water was modeled by applying a diffusion kernel, $\mathbf{D}_{\mathbf{H} 2 \mathbf{O}}$, on the magnetization lattices as already proposed [22,23]:

$$
\mathbf{M}_{\mathbf{i}}=\mathbf{M}_{\mathbf{i}} \otimes \mathbf{D}_{\mathbf{H} 2 \mathbf{O}} \quad \text { with } \quad \mathbf{D}_{\mathbf{H} 2 \mathbf{O}} \propto \frac{1}{\sigma^{2}} e^{-\frac{x^{2}+y^{2}}{2 \sigma^{2}}}
$$

where the mean square displacement of a water molecule is $\left\langle x^{2}+y^{2}\right\rangle=2 \sigma^{2}=4 A D C \delta t$ (in 2D, [22]) and $i$ stands for $\perp$ or $\|$.
The convolution with the kernel modeled the probability of the spins to move to a different location during the time $\delta t$. Due to the finite extension of the lattice, the kernel was subsequently normalized to unity. We assumed that water diffused freely within the plane and between compartments. The convolution was performed using the FFT algorithm.

(c.5) MR sequence. A saturation-recovery sequence with a multi gradient-echo acquisition scheme was simulated. At each echo time $T_{E}$, the MR signal was sampled by summing the transverse magnetization $\mathbf{M}_{\perp}$ across the lattice. At $t$ modulo $T_{R}, \mathrm{RF}$ excitation pulse, characterized by flip angle $\alpha$ and phase $\phi$, was applied on the magnetization lattices. For spin-echo case, a $\pi \mathrm{RF}$ pulse was applied at $T_{E}^{S E} / 2$.

Unless mentioned otherwise, the parameter values used in the simulation were as follows. The static magnetic field $B_{0}$ was set to $4.7 \mathrm{~T}$, the time step $\delta t$ to $0.5 \mathrm{~ms}$ and the total duration of the experiment $T_{F}$ to $900 \mathrm{~s}$. The geometry modeled was a $70 \times 70 \mu \mathrm{m}^{2}$ plane sampled with $Q^{2}=560^{2}$ elements (lattice point size $\left.0.015 \mu m^{2}\right) . N=5$ vessels of radius $R=3 \mu m$ were generated, filling up $B V f=2.9 \%$. These values match in vivo measurements in healthy tissue [24,25]. In vivo measurements made in rodents provided the AIF shape, $c_{v}(t)$ [26]. Relaxation properties of the CA matched Gd-chelate ones: $r_{1}=3.3 s^{-1} . m M^{-1}$, $r_{2}=4.1 s^{-1} \cdot m M^{-1}$ (data from Guerbet, France). The initial relaxation rates were, in the vessels, $R_{1}^{0, v}=0.582 s^{-1}$ [27], $R_{2}^{0, v}=200 s^{-1}$ (higher than previously reported value [28]), in the tissue, $R_{1}^{0, t}=0.769 s^{-1} \quad[29], \quad R_{2}^{0, t}=16 s^{-1} m s$. We used $\chi_{m}=0.027$ ppm. $m M^{-1}$ for the molar susceptibility of the CA $[30]$ and $\Delta \chi_{\text {blood-tissue }}=0.0422 \mathrm{ppm}(\mathrm{SO2}=60 \%$ and $[\mathrm{Hct}]=40 \%$ at $4.7 T$ [31]) (CGS units). The water diffusion was set to $760 \mu \mathrm{m}^{2} \mathrm{~s}^{-1}$ [32]. $T_{R}$ was set to $500 \mathrm{~ms}, \alpha$ to $90^{\circ}$ and $\phi$ to $0^{\circ}$ [26].

As a reference for $k_{p e}$, we used $k_{0}=1.83 \cdot 10^{-3} s^{-1}$ and $k_{1}=4.83 \cdot 10^{-3} s^{-1}$, values reported in [26] for healthy muscle tissue and for tumor tissue. An illustration of the shape of the AIF is presented in Fig. 3b. For $D_{C A}$, we used $D_{0}=46 \mu \mathrm{m}^{2} \mathrm{~s}^{-1}$ which correspond to the coefficient of diffusion for Gd-DOTA measured in rat brain [33]. The free diffusion of Gd-DOTA, $D_{\text {free }}=485 \mu \mathrm{m}^{2} \mathrm{~s}^{-1}$, has also been reported [33].

\section{Validation}

Contrast Agent Diffusion. To evaluate our kernel based approach used to model constrained diffusion, we compared our results to a 2D Monte-Carlo (MC) simulation. In the MC approach, elastic rebounds of CA on the surface of obstacles were considered [34]. Diffusion with both approaches was simulated within the same geometry, $N=5$ and $R=3 \mu \mathrm{m}$. The apparent diffusion coefficient of $\mathrm{Gd}$ was set to $D_{0}$. Due to the extensive computation time of the MC approach, we simulated the diffusion process during $3 \mathrm{~s}$. The CA was initially positioned at the center of the grid: $1 \mathrm{mM}$ in a single pixel for the kernel simulations, 500,000 particles randomly spread within the same pixel for the $\mathrm{MC}$ simulations. To allow comparison between the two approaches, the total amount of matter of each approach was equalized afterwards.

Blood flow and GA Permeability. To investigate the validity of the approach used to model the permeability, we fitted the concentration profiles with the classic modified Tofts model [8] using a nonlinear Levenberg-Marquardt algorithm.

$$
[C A](t)=B V f . c_{v}(t)+k_{p e} \cdot v_{e} \int_{0}^{t} c_{v}(\tau) e^{-k_{p e}(t-\tau)} d \tau
$$




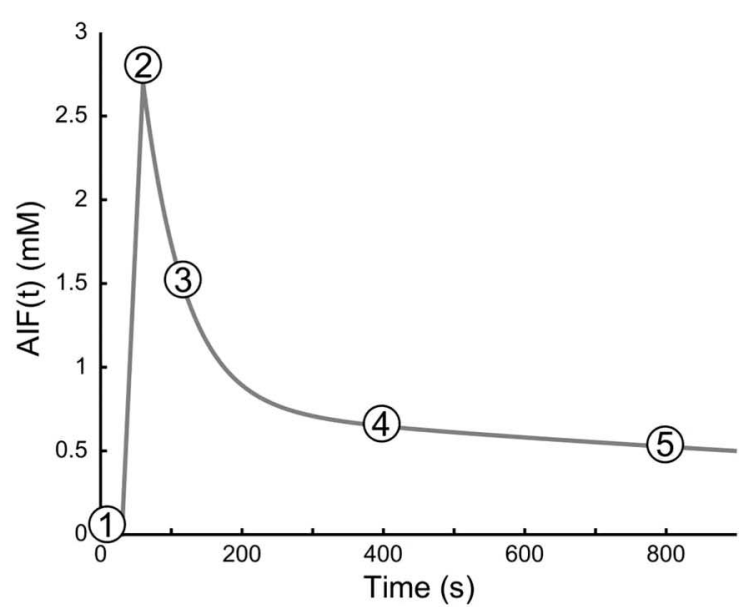

(1)

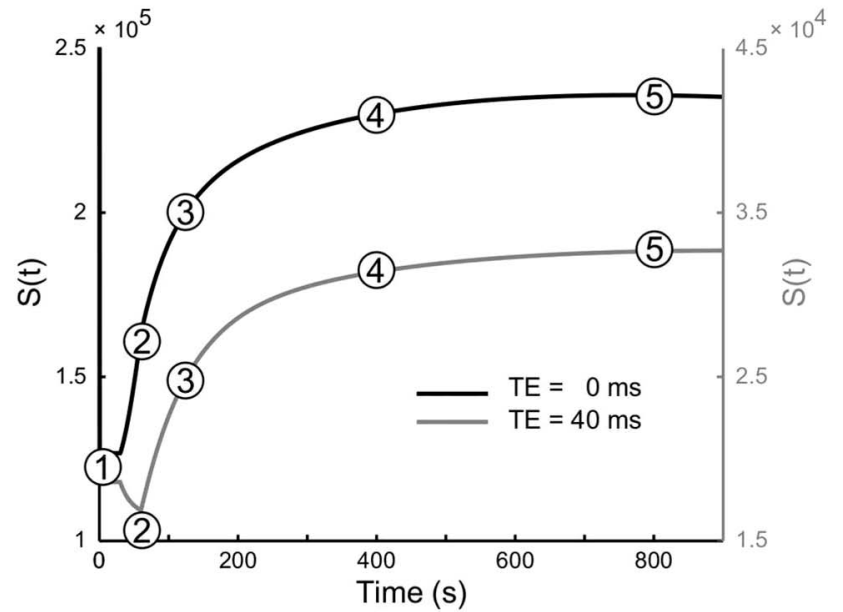

(3)
(4)
(5)
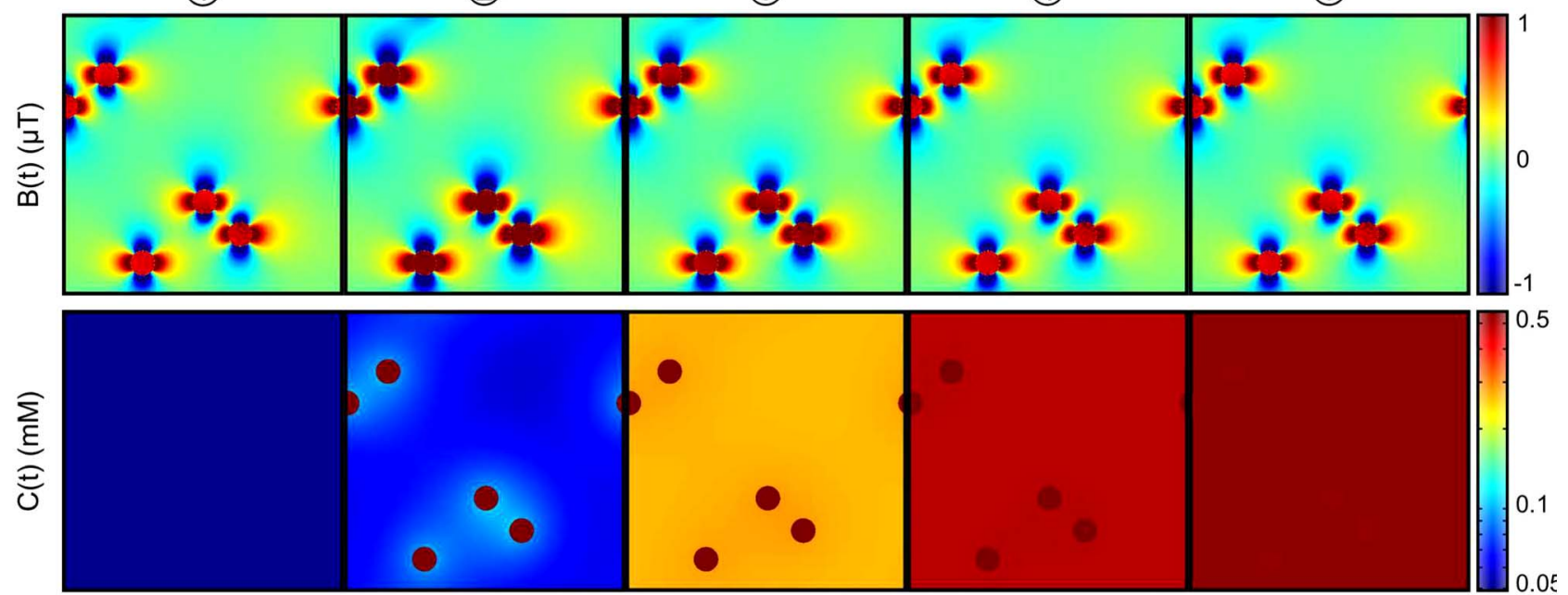

Figure 3. Illustration of the evolution of the concentration of CA. CA concentration in the vessels $c_{v}(t)$ (a) and the corresponding MR signal $S(t)$ (b). $S(t)$ is simulated for 2 echo times: $T_{E}=0 \mathrm{~ms}$ (black) and $T_{E}=40 \mathrm{~ms}$ (grey). The change in CA concentration $\mathbf{C}(t)$, represented by the lattices, and in the magnetic field perturbations $\Delta \mathbf{B}(t)$ are presented at five times points labeled (1) to (5). For this longer echo time, one can observe the competition between the susceptibility effect which decreases the signal (point (2)) and the enhancement produced by the $R_{1}$ relaxation effect of the CA which extravasates into the tissue (points (3) to (5)). At the last simulation time point $\left(t=T_{F}\right)(5), c_{v}(t)$ is lower than $[C A](t)$ (not shown) and the concentration in the extravascular space begins to decrease. Note the log scale for $\mathbf{C}(t)$ introduced for sake of clarity. doi:10.1371/journal.pone.0057636.g003

We eventually compared the estimate of $k_{p e}, k_{p e}^{F i t}$, with the theoretical $k_{p e}, k_{p e}^{T h}$, introduced as an input of the simulation at step (b.2). We denoted $B V F^{F i t}$ the estimate of $B V f$.

Magnetic Field Perturbations. To validate the 2D technique used to compute the magnetic field perturbations, we balanced the corresponding 3D model (magnetic field perturbations induced by isotropic distributed cylinders in space and orientation [35]) with 3 different 2D approaches: 1 unique vessel in $1 B_{0}$ orientation, $\mathrm{N}$ vessels in $1 B_{0}$ orientation, $\mathrm{N}$ vessels in $3 B_{0}$ orientations. For each approach, we simulated and averaged the free induction decays (FID) provided by a set of 70, randomly obtained, geometries (only 1 geometry for the first 2D approach, and we estimated $R_{2}^{G E}$ by a mono-exponential function fitted to the mean FID with a nonlinear Levenberg-Marquardt algorithm. Each geometry had the same properties: $B V f=2.9 \%, R=3 \mu \mathrm{m}$, $N=1$ or $N=5$ for the 2D approach, $N=12$ for the 3D approach. To match the conditions described by [35], the magnetic susceptibility difference between the vessels and the tissue was set to $\Delta \chi=0.231 \mathrm{ppm}$.
Relaxation changes vs vessel radius. To further investigate the validity of the proposed approach, we simulated the dependency of the gradient-echo (GE) and spin-echo (SE) relaxation rates $\left(\Delta R_{2}^{G E}\right.$ and $\left.\Delta R_{2}^{S E}\right)$ with the radius of the vessels as previously described [36-38]. For a given vessel radius, we randomly generated a set of 10 different geometries with $N=5$ vessels that occupy $2 \%$ of a plane. To match these constraints, the plane size was adjusted to the vessel radius. Eighteen vessel radii were simulated, between 1 and $100 \mu \mathrm{m}$. To match the conditions used by Boxerman et al. [37], the diffusion of water was set to $1000 \mu \mathrm{m}^{2} . \mathrm{s}^{-1}, B_{0}$ to $1.5 T$ and $\Delta \chi$ to $0.1 \mathrm{ppm}$. The MR signal was simulated at $T_{E}=60 \mathrm{~ms}$ for gradient-echo type experiment and at $T_{E}^{S E}=100 m s$ for spin-echo type experiment. The relaxation rates were computed with Eq.[15]:

$$
\Delta R_{2}^{G E}, \Delta R_{2}^{S E}=-\frac{1}{T_{E}} \ln \left(\frac{S\left(T_{E}\right)}{S(0)}\right)
$$


Impact of Diffusion, Permeability and Echo Time

The concentration $c(t)$ was derived from the MR signal $S(t)$ as described in [26]:

$$
c(t)=\frac{1}{r_{1}}\left(R_{1}(t)-R_{1}^{0}\right)
$$

with

$$
R_{1}(t)=-\frac{1}{T_{R}} \ln \left(1-\frac{S(t)}{S(0)}\left(1-\exp \left(-R_{1}^{0} \cdot T_{R}\right)\right)\right)
$$

This equation is valid only under certain assumptions $\left(T_{R} \gg T_{2}^{*}\right.$, $T_{R} \gg T_{2}$ (no stimulated echoes), $T_{E} \ll T_{2}^{*}, \pi / 2$ flip angle, no inflow, etc.). We then fitted either $[C A](t)$ or $c(t)$ with the bi-compartment model (Eq.[14]) to derive the errors on estimated permeability constant: $\left(k_{p e}^{F i t}-k_{p e}^{T h}\right) / k_{p e}^{T h}$.

\section{Results}

For sake of simplicity, unless mentioned otherwise, the results presented in this study are obtained with no cells positioned in the extravascular space and in the limit of high flow $(F . \delta t>B V f)$, i.e. $c_{v}(t)=c_{a}(t)$.

As an illustration, Fig. 3 shows the changes in $\mathbf{C}(t)$ and $\mathbf{\Delta B}(t)$ throughout the simulation together with the input $A I F(t)$ and the output $S(t)$ at two gradient-echo times: $T_{E}=0 \mathrm{~ms}$ and $T_{E}=40 \mathrm{~ms}$, with $k_{p e}=k_{0}$ and $D_{C A}=D_{0}$.

\section{Validation}

Contrast Agent Diffusion. Fig. 4a presents the geometry lattice $\mathbf{G}_{\mathbf{v}}$ and the injection site. Fig. $4 \mathrm{c}$ and Fig. 4d show the diffusion maps obtained with the MC and the kernel approaches respectively. Due to low SNR for the MC approach, the displayed lattices were smoothed to ease their visualization. Fig. 4b shows the correlation graph between the two approaches. The two maps are in good agreement $\left(R^{2}=0.994\right)$ with a slightly faster diffusion process observed in the MC approach (slope $=0.992$ ).

Blood flow and CA Permeability. The results of this section were obtained with $\delta t=25 \mathrm{~ms}$. Fig. 5a shows the concentration profiles obtained for different blood flow values when $k_{p e}=0$. When $F \delta t \geq B V f$, the flow is high enough to renew the blood volume at each time step $\delta t$ and $c_{v}(t)=c_{a}(t)$. When $F . \delta t<B V f$, i.e $F<1.2 s^{-1}$ in our experimental conditions, the concentration profiles are modified accordingly to the dilution of the arterial input within the vascular compartment. This situation corresponds to a single compartment model with a mono-exponential residue function with characteristic time $B V f / F$.

Fig. 5b illustrates the time courses of $c_{a}(t)$ and $[C A](t)$ when $k_{p e}=k_{1}$ for different blood flows $F: 3.10^{-3}-15.10^{-3} s^{-1}$ (equivalent to $19-90 \mathrm{~mL} / \mathrm{min} / 100 \mathrm{~mL}) . c_{a}(t)$ is an input to the simulation (step b.1) and $[C A](t)$ is the output of block b. As depicted by the concentration curve shapes $[C A](t)$, the uptake of CA is limited when the blood flow decreases.

We simulated the change in $[C A](t)$ for 4 permeability values, 4 blood flows and with $D_{C A}=D_{\text {free }}$ (to mimic an infinite diffusion coefficient as assumed by a bi-compartmental model). The Tofts model properly fits the data for the range of permeabilities and blood flow simulated (Fig. 5b). Fig. 5c presents the results of the estimated parameter $k_{p e}^{F i t}$. For high flow, a linear correlation between $k_{p e}^{F i t}$ and $k_{p e}^{T h}$ is observed $\left(R^{2}=0.999994\right)$. The slope is slightly lower than 1 and demonstrates that $k_{p e}^{F i t}$ underevaluates $k_{p e}^{T h}$ when the CA diffusion is slightly constrained $\left(D_{C A}<\infty\right)$. As already reported, for lower blood flow values, the CA leakage is limited by the inlet and the model fails to distinguish the blood flow from the permeability [8].

Magnetic Field Perturbations. The different voxel configurations are displayed on Fig. 6a-d. Fig. 6e presents the mean normalized FID obtained with each approach. The single vessel approach results in a very short decay $\left(1 / R_{2}^{G E}=7.5 \mathrm{~ms}\right)$ whereas the $\mathrm{N}$ vessels averaged over 3 directions approach $\left(1 / R_{2}^{G E}=18.3 \mathrm{~ms}\right)$ presents a decay similar to what is obtained with the $3 \mathrm{D}$ approach $\left(1 / R_{2}^{G E}=18.4 m s\right)$.

Relaxation changes vs vessel radius. Fig. 7 shows the dependence of $\Delta R_{2}^{G E}$ and $\Delta R_{2}^{S E}$ on the vessel radius. The results of our simulation are in very good agreement with similar works obtained with other approaches $[37,38]$. We also observe that the standard deviation is larger for the GE experiments than for the SE experiments.

\section{Impact of Diffusion, Permeability and Echo Time}

We simulated a DCE experiment with various CA diffusion coefficients $\left(D_{0} / 4, D_{0} / 2, D_{0}, 2 \times D_{0}, 4 \times D_{0}, 10 \times D_{0}, D_{\text {free }}\right)$, various permeabilities to $\mathrm{CA}\left(k_{0}, k_{0} / 2, k_{1}\right)$ and various $T_{E}$ (20 echoes, $\Delta T_{E}=2 m s$.

Fig. 8 illustrates $S(t)$ obtained for different $D_{C A}$ values and $k_{p e}^{T h}$ values. As expected, $k_{p e}^{T h}$ strongly impacts the shape of $S(t)$ (Fig. 8a). Conversely, the CA diffusion coefficient has a small impact on the shape of $S(t)$. The highest deviation appears when the CA concentration in the vessels is maximum.

Fig. 9a presents the permeability estimates derived from $[C A](t)$. The higher the permeability, the larger the error on $k_{p e}^{F i t}$. The faster the CA diffusion, the smaller the error on $k_{p e}^{F i t}$. For the range of CA diffusion coefficients used in this simulation, the error never exceeded $10 \%$. The highest error was obtained for the lowest $D_{C A}$ coefficient (Fig. 9a). This means that when the CA leaves slowly the vessel periphery, the vessel permeability is underestimated. As expected, this underestimation becomes smaller as $D_{C A}$ increases.

When the permeability estimates are derived from $S(t)$ at $T_{E}=0 m s$, the behavior of the error on $k_{p e}^{F i t}$ remains unchanged (Fig. 9b). For high $D_{C A}$ and low $k_{p e}^{T h}$, the errors become negative, in agreement with the result observed on Fig. 5b (slope smaller than one). The error on the permeability estimate remaines below $5 \%$. However, a difference between the permeability estimates obtained from $[C A](t)$ and $S(t)$ appears for high $D_{C A}$ values : the error is independent of $k_{p e}^{T h}$ when one analyzes $[C A](t)$ and becomes a function of $k_{p e}^{T h}$ when one analyzes $S(t)$. This difference may be ascribed to the $R_{1}^{0}$ value used in Eq. [17] which assumes a slow exchange regime across the vessel wall (the effective $R_{1}^{0}$ could not be computed since the water permeability was not controlled for in our model).

Fig. 10a shows the variation of the error on $k_{p e}^{F i t}\left(k_{p e}^{F i t}\right.$ as a function of $T_{E}$ for different $k_{p e}^{T h}$ and $\left.D_{C A}\right)$. For short $T_{E}$, the error is minimum and the larger the CA diffusion, the lower the error as already observed in Fig. 9b. For $k_{p e}^{T h}=k_{1}$, the error increases with $T_{E}$. For $k_{p e}^{T h}=k_{0}$ and $k_{p e}^{T h}=k_{0} / 2$ the evolution of the error is no longer proportional to $T_{E}$ and ranges from $25 \%$ (for $k_{p e}^{T h}$ ) up to $200 \%$ (for $B V f^{\text {Fit }}$ ). Fig. 10b shows $B V f^{\text {Fit }}$ as a function of $T_{E}$ for different $k_{p e}^{T h}$ and $D_{C A}$. This error is the largest for long $T_{E}$. This deviation from the input $B V f$ is ascribed to the post-processing: 
a)

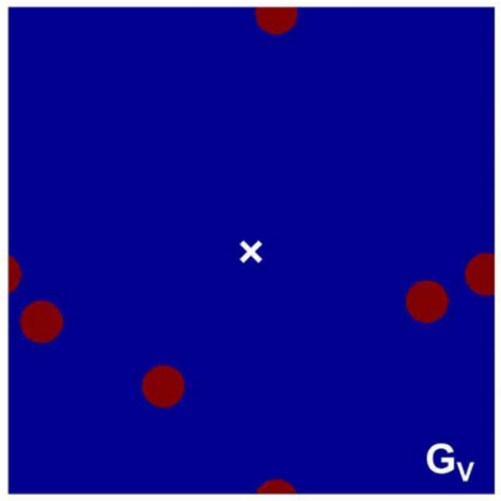

c)

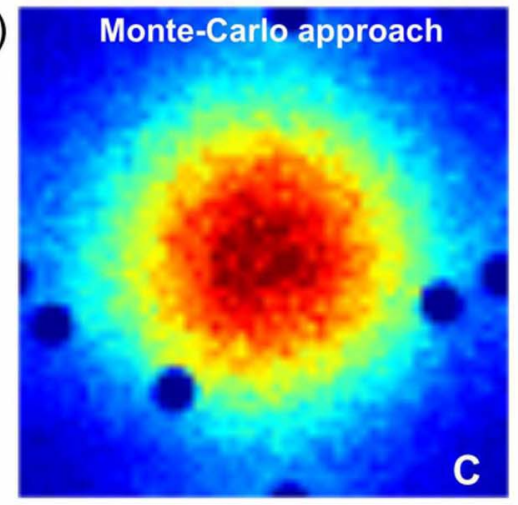

b)

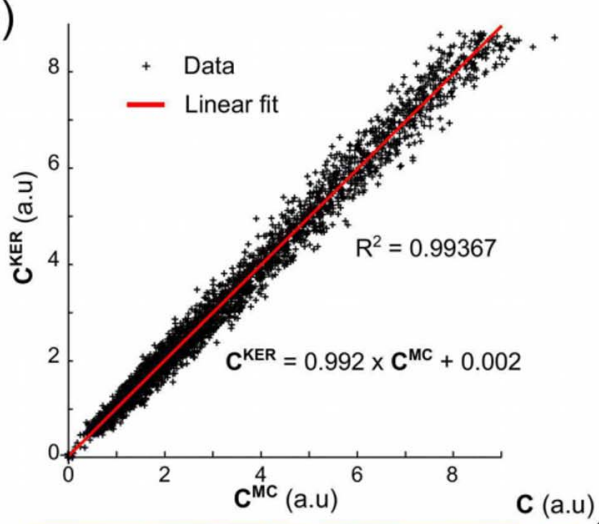

d)

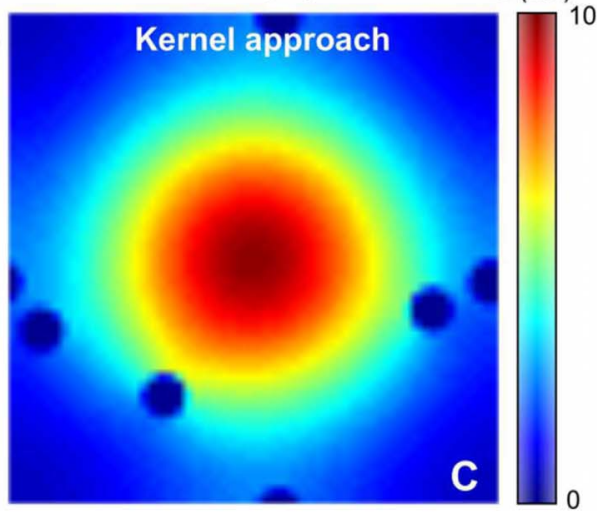

Figure 4. Comparison between MC approach and kernel based approach for modeling the CA diffusion. (a) Geometry used, $\mathbf{G}_{\mathbf{v}}$. The white cross indicates where the CA was initially placed. (b) Spatial correlation plot between $\mathbf{C}$ obtained via the convolution with a diffusion kernel and $\mathbf{C}$ obtained with the MC approach after normalization. (c-d) Final maps of CA concentration, $\mathbf{C}$, for the MC approach $\left(C^{M C}\right)$ and the kernel approach $\left(C^{K E R}\right)$, respectively (smoothed and undersampled to a $64 \times 64$ lattice).

doi:10.1371/journal.pone.0057636.g004

the $T_{2}^{*}$ effects which are maximum at peak concentration are not taken into account in Eq.[17] (Fig. 8a). At this echo time, the data processing yields an erroneous estimate of $B V f$ (Fig. 10b). For $k_{p e}^{T h}=k_{0} / 2$, we note that $B V f^{F i t}$ appears sensitive to $D_{C A}$ for long echo times.

The effect of the echo time on the permeability error is much stronger than that of $D_{C A}$. Going from 0 to $10 \mathrm{~ms}$ may increase
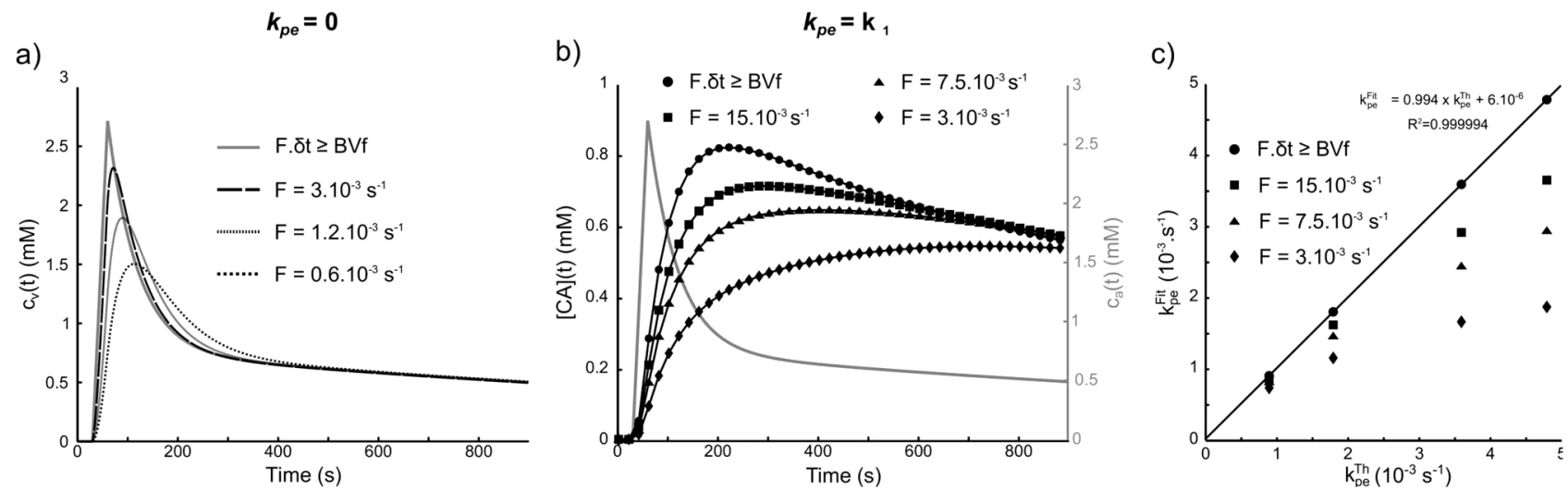

Figure 5. Concentration profiles for various blood flows and permeabilities to CA. (a) Concentration of CA in the vascular compartment, $c_{v}(t)$, as a function of time for impermeable vessel wall $\left(k_{p e}^{T h}=0\right)$ and different blood flow values. (b) Time course of $c_{a}(t)$ and $[C A](t)$ for $k_{p e}^{T h}=k_{1}$ and different blood flow values. $[C A](t)$ is plotted every 20 s to ease readability. The plain black lines represent the fit obtained with the Tofts model (Eq.[14]). Note the difference in scale for the arterial input function, $c_{a}(t)$. (c) Plots of the estimated permeability coefficient $k_{p e}^{F i t}$ and the input value $k_{p e}^{T h}$ for different blood flows and permeabilities to CA. A linear fit is obtained in the case of high flow $(F . \delta t \geq B V f)$. For lower blood flows, the model failed to distinguish the flow from the permeability and $k_{p e}^{F i t}$ is underestimated.

doi:10.1371/journal.pone.0057636.g005 
a)

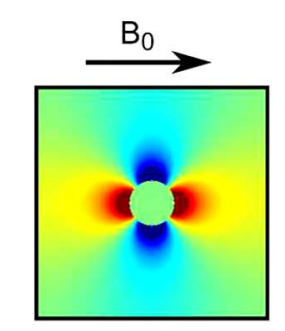

c)

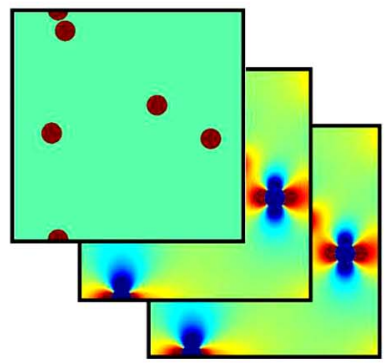

b)

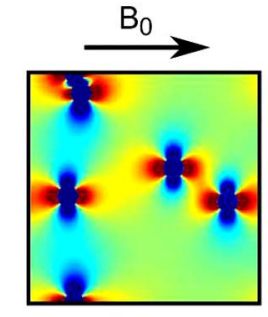

d)

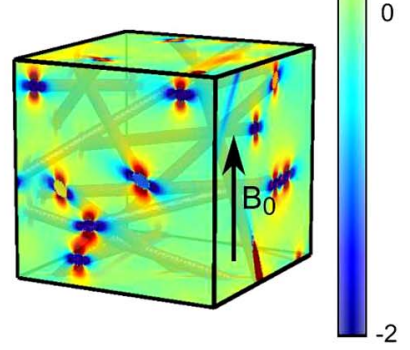

$\Delta \mathrm{B}(\mu \mathrm{T})$

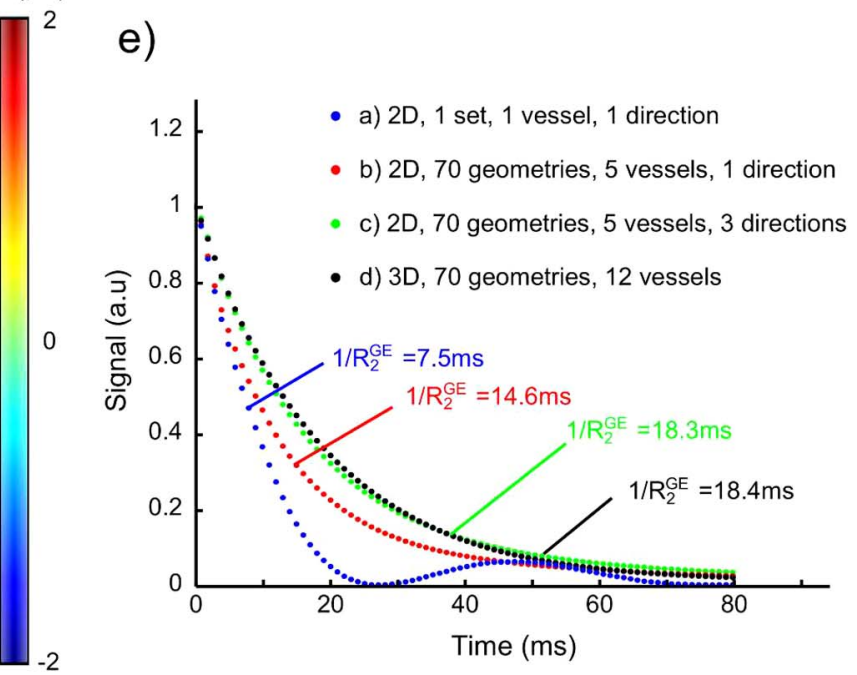

Figure 6. Impact of various magnetic field computations on the FID simulation. (a) 1 vessel in $1 B_{0}$ orientation (b) $\mathrm{N}$ vessels in $1 B_{0}$ orientation (c) $\mathrm{N}$ vessels in $3 B_{0}$ orientations (d) $\mathrm{N}$ vessels in 3D. The vessel arrangement is presented in 3D and for display, the magnetic field perturbation is only presented on each face of the cube but is computed in 3D. (e) Normalized FID for approaches (a)-(d) (averaged across the geometries for approaches $(b-d)$ ).

doi:10.1371/journal.pone.0057636.g006

the error from $2 \%$ to $8 \%$. This impact is even more serious on the $B V f$ estimate: the same increase in echo time yields an underestimation of $B V f^{F i t}$ by a factor 2. This is likely related to the competition which occurs between the varying susceptibility gradients at the vessel wall and the increasing $R_{1}$ in the extravascular space. This balance depends on $T_{E}$ and on $D_{C A}$.

\section{Versatility}

Realistic microvasculature network. To illustrate the potential of the proposed simulation tool, Fig. 11 shows the results obtained with a vasculature network extracted from a biological tissue. The geometry was acquired with a two-photon laser

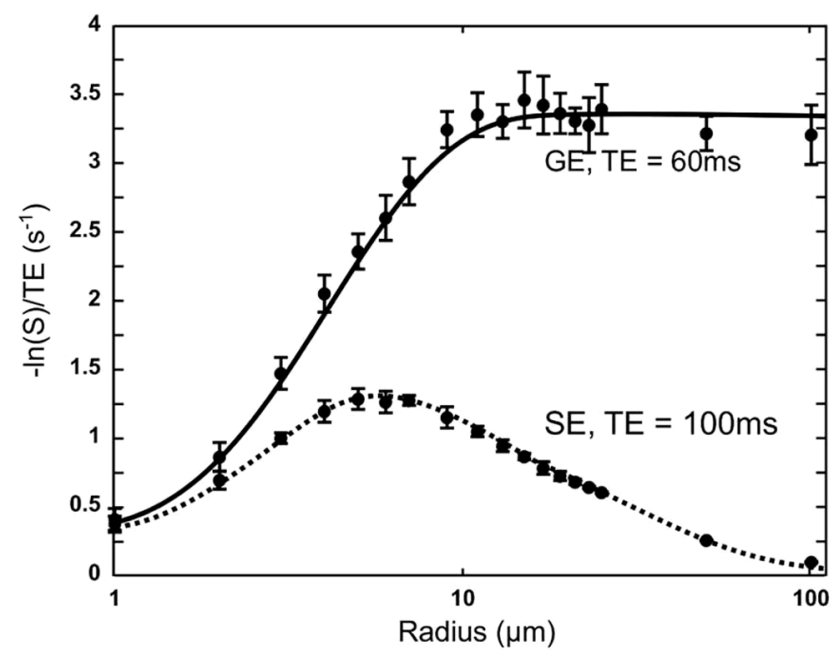

Figure 7. Vessel radius dependence of $\Delta R_{2}^{G E}$ and $\Delta R_{2}^{S E}$. Parameters values are $B V f=2 \%, A D C=1000 \mu m^{2} s^{-1}, B_{0}=1.5 T$ and $\Delta \chi=0.1 \mathrm{ppm}$. Mean $\pm \sigma$ across 10 geometries. The data presented here are in excellent agreement with those reported in [37].

doi:10.1371/journal.pone.0057636.g007 scanning microscope with $5 \mu \mathrm{m}$ z-step [35]. Image size was $560 \times 560$ pixels with a field of view of $300 \times 300 \mu \mathrm{m}$. Morphological processing (erosion/dilatation) were used to fill holes in the vessels and to remove isolated pixels. A threshold was used for image segmentation (blood/tissue). A representation of the vascular network as the $2 \mathrm{D}$ binary lattice $\mathbf{G}_{\mathbf{v}}$ was eventually obtained. In Fig. 11 , the blood vessels occupy $B V f=1.92 \%$ of the surface. The simulation was performed with $k_{p e}^{T h}=k_{0}$ and $D_{C A}=D_{0}$. The lattices $\mathbf{C}(t)$ (Fig. 11a) and $\mathbf{\Delta} \mathbf{B}(t)$ (Fig. 11b) are presented at $t=T_{F}$. The concentration profiles derived from the simulated MR signal using Eqs.[16-17] are shown for 3 different $T_{E}$ on Fig. 11c. The Toft model was eventually fitted to the data. The estimates were in agreement with the input values when $T_{E}=0 m s\left(k_{p e}^{F i t}=0.017 s^{-1}, B V F^{F i t}=3.0 \%\right)$ and biased for longer $T_{E}$ (at $T_{E}=40 m s, k_{p e}^{F i t}=0.017 s^{-1}, B V F^{F i t}=-1.6 \%$ ).

Porosity of the extravascular space. Fig. 12 presents the results obtained with cells positioned in the extravascular space. Vessel geometry was the same as displayed on Fig. 3. The simulation was performed with $\Pi=20 \%, \quad k_{p e}^{T h}=k_{0}$ and $D_{C A}=D_{\text {free }}$. The lattices $\mathbf{C}(t)$ (Fig. 12a) and $\mathbf{\Delta} \mathbf{B}(t)$ (Fig. 12b) are presented at $t=t_{F}$. The concentration profiles derived from the simulated MR signal using Eqs.[16-17] are shown for 3 different $T_{E}$ on Fig. 12c. The Toft model was also fitted to the corresponding data and the results were in agreement with previously described ones (at $T_{E}=0 \mathrm{~ms}$, $k_{p e}^{F i t}=0.018 s^{-1}, B V F^{F i t}=3.33 \%$ ) and biased for longer $T_{E}$ (at $\left.\left.T_{E}=40 m s, k_{p e}^{F i t}=0.023 s^{-1}, B V F^{F i t}=-1.7 \%\right)\right)$. The difference in the concentration scale is due to the reduced interstitial volume. For long echo times, the additional magnetic field perturbations that arose at the cell membranes reduce the enhancement effect and inflection points can be noticed in the concentration profiles (Fig. 12a at $T_{E}=40 \mathrm{~ms}$ and $T_{E}=70 \mathrm{~ms}$ ). 

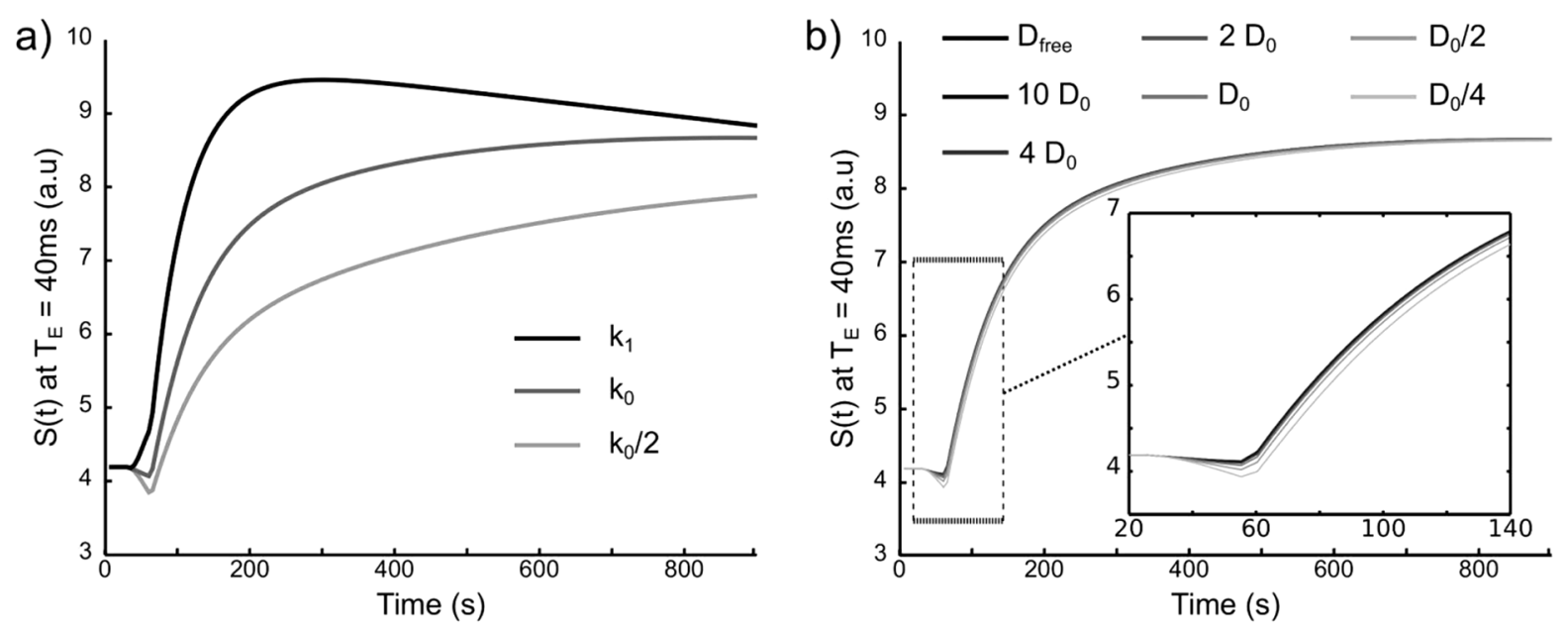

Figure 8. Change in the MR signal for different $k_{p e}^{T h}$ and $D_{C A}$ values. (a) $\mathrm{S}(\mathrm{t})$ at $T_{E}=40 \mathrm{~ms}$ for $3 k_{p e}^{T h}$ values: $k_{1}, k_{0}$ and $k_{0} / 2$ with $D_{C A}=D_{0} / 4$. (b) $\mathrm{S}(\mathrm{t})$ at $T_{E}=40 \mathrm{~ms}$ for $7 D_{C A}$ values: $D_{0} / 4, D_{0} / 2, D_{0}, 2 D_{0}, 4 D_{0}, 10 D_{0}$ and $D_{\text {free }}$ with $k_{p e}^{T h}=k_{0}$.

doi:10.1371/journal.pone.0057636.g008

\section{Discussion}

In this study we proposed a tool to simulate a DCE-MRI experiment with intravenous CA injection and altered BBB. The simulation tool takes into account the blood flow, the CA extravasation via a controlled vessel wall permeability, the CA diffusion within the extravascular space, the water diffusion, the vessels and CA susceptibility effects together with the relaxivity effects. More realistic models for flow such as plug-flow or not well-mixed blood compartment are disregarded. Each important step of the simulation was validated and we discuss the deviation observed in the following. At various echo times, the impact of the CA diffusion on the permeability estimate was investigated in the limit of high flow. The versatility of the algorithm was finally demonstrated with geometries based on a realistic vascular network or space constrained by cells. The results obtained were in agreement with previously described in vivo experiments.

\section{Fourier based approaches}

The simulation benefits from the extensive used of the FFT for the computation of the magnetic field perturbations, the diffusion of the CA and the diffusion of the water molecules. In addition to the gain in speed compared to classic convolution algorithms, we take advantage here of the intrinsic properties of the discrete Fourier transform: the spatial sampling yields a spatial periodization. Thereby, with the diffusion kernel approach, the CA which leaves the lattice on one edge comes back on the opposite edge, as if the lattice was surrounded by similar lattices. Consequently, our model takes into account the contribution of the CA movements due to diffusion and arriving from adjacent voxels [39]. Interestingly, this approach, which is also relevant in $3 \mathrm{D}$, can be extended to any geometries with obstacles such as extravascular cells (Fig. 12). This property also applies to the magnetization carried by the water molecules, which freely diffuse within the voxel, and to the magnetic field computation. Note also that the

\section{From $[\mathrm{CA}](\mathrm{t})$}

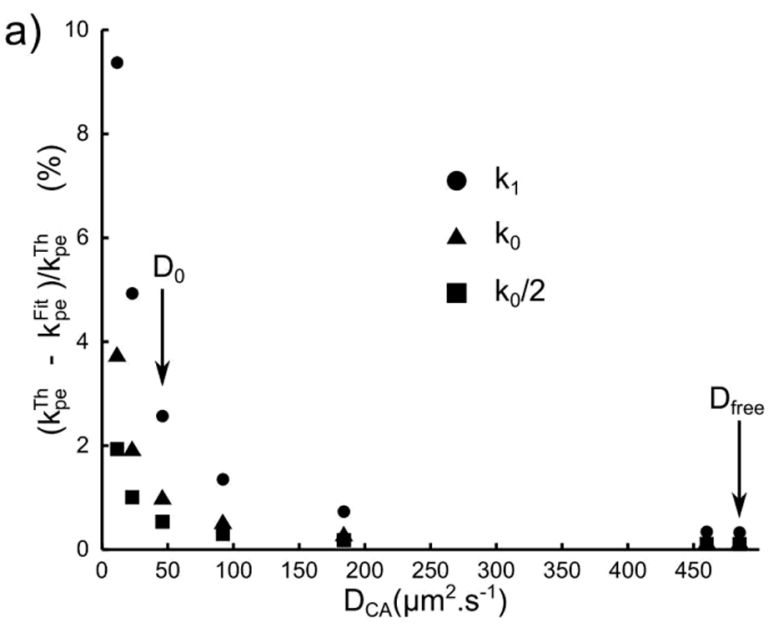

From $S(t)$

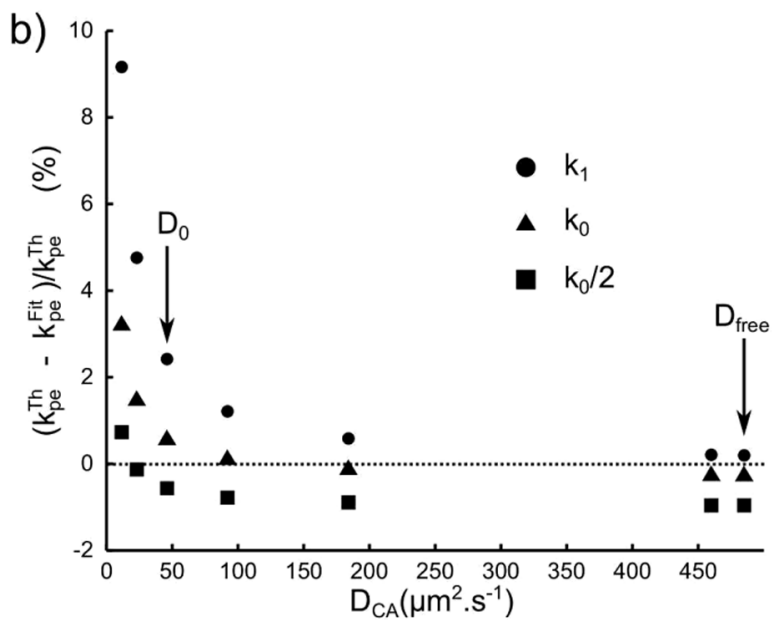

Figure 9. Error on the permeability estimate. When modeling the outputs of blocks b and c with Eq.[14] for various $k_{p e}^{T h}$ and $D_{C A}$ values: (a) Error on $k_{p e}^{T h}$ when modeling $[C A](t)$. (b) Error on $k_{p e}^{T h}$ when modeling $\mathrm{S}(\mathrm{t})$ for $T_{E}=0 m s$ with Eqs.[16-17]. doi:10.1371/journal.pone.0057636.g009 

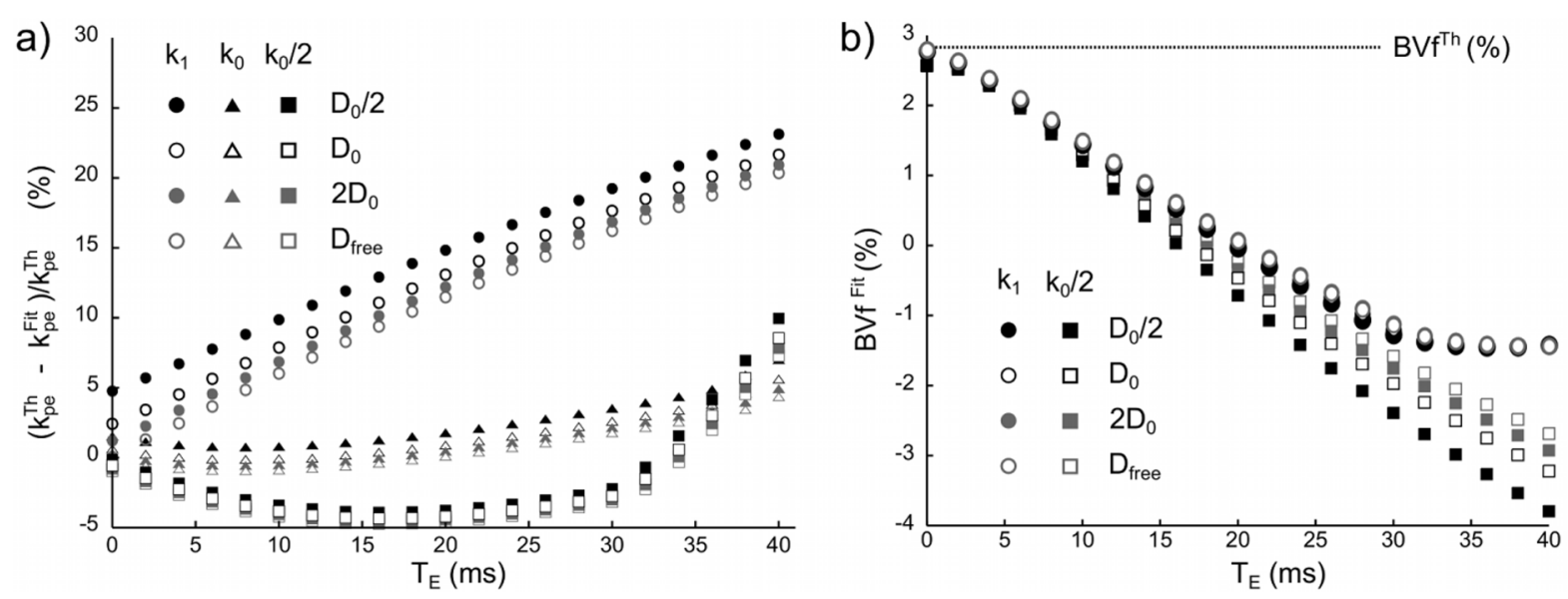

Figure 10. Impact of the echo time on the estimation of $k_{p e}^{T h}$ and $B V f$. (a) Evolution of the error on the parameter $k_{p e}^{T h}$ estimated from $S(t)$ at different $T_{E}$ for various $D_{C A}$ and various $k_{p e}^{T h}$. (b) Evolution of the parameter $B V f^{F i t}$ estimated from $S(t)$ at different $T_{E}$, for various $D_{C A}$ and for $k_{p e}^{T h}=k_{0} / 2$ or $k_{1}$.

doi:10.1371/journal.pone.0057636.g010

Gibbs artifacts at the border were here avoided thanks to the periodization of the $2 \mathrm{D}$ vasculature. These artifacts usually arose at the border of $3 \mathrm{D}$ voxel simulation and compel to reject the spoiled outer volume of the $3 \mathrm{D}$ lattice for the signal computation.

\section{CA diffusion}

The main asset for using the kernel approach to model diffusion is its computational efficiency. The diffusion process appeared however slightly slower than with the MC approach. This reduction is related to the use of the weighted lattice $\mathbf{W}_{\mathbf{C A}}$ : the amount of $\mathrm{CA}$ that diffuses from a pixel located within the interstitium to pixels located inside a vessel or a cell is sent back to it initial position. This differs from the MC approach where elastic collisions are considered. Thus, the CA diffusion modeled with the kernel approach is more hindered at the vicinity of vessels and cells than with the MC approach. From a physiological point of view, note that it has been proposed that diffusion near cells could be reduced beyond elastic collision due to electrostatic interactions with cell membranes [40,41].

The kernel must also be narrow enough to avoid 'jumps'. When the kernel width, $\sigma$, is larger than the characteristic obstacle size, $s_{m}$, the CA diffusion becomes blind to obstacles. To avoid this behavior, one must observe $\sigma<s_{m}$. This 'no-jump' condition was respected in this study: $\sigma=\sqrt{2 D_{\text {free }} \delta t} \approx 0.5 \mu m$ and $s_{m}=2 . R=6 \mu m$. This condition may be fulfilled for a large range of $D_{C A}$ values and obstacle sizes by adapting $\delta t$ accordingly.

It is also worth noting that, by adding kurtosis or skewness terms to the kernel, the kernel approach offers the opportunity to model non-Gaussian diffusion and/or active transport.

\section{Magnetic field perturbations}

The approach used in this study to compute the magnetic field perturbations is based on the Fourier transform of the magnetic susceptibility lattice [20,21]. Most works employed the analytical form of the magnetic field perturbations generated by an infinite straight cylinder $[23,36,37,42,43]$. However, this latter technique, computationally efficient, fails at computing smooth susceptibility variations and more realistic vasculatures which vessel calibers, vessel densities and tortuosity may be significantly modified as in tumors $[2,24,44]$. Another approach, based on the perturbation produced by a single pixel convolved with a geometry lattice $\left(\mathbf{G}_{\mathbf{v}}\right.$ in our study) [38], has been proposed. This latter approach is
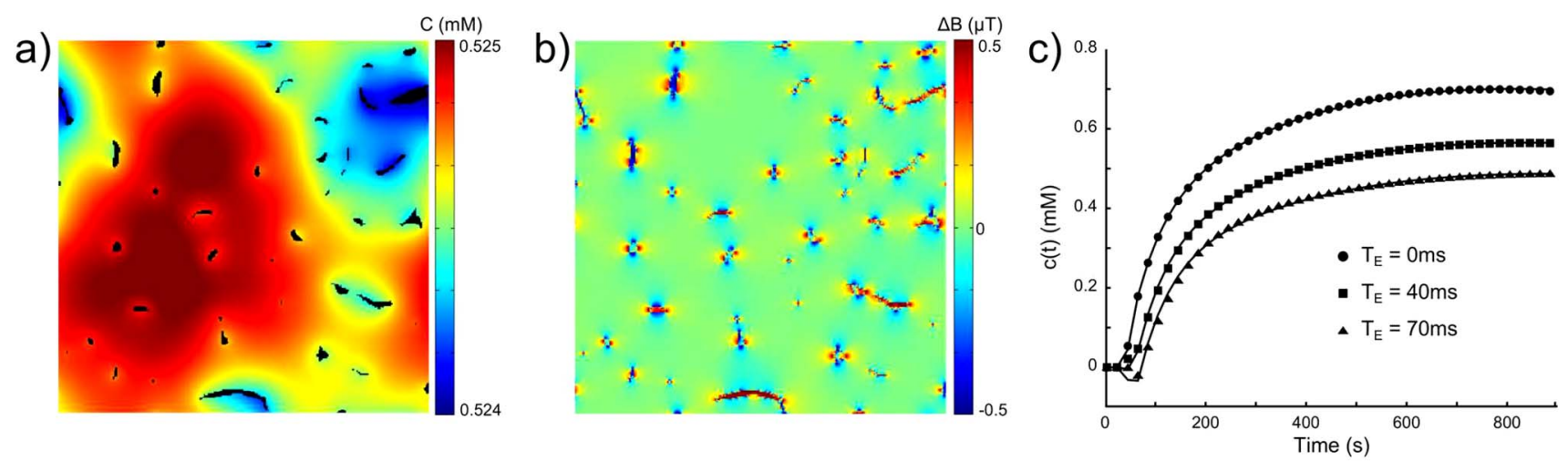

Figure 11. Example of the simulation with a vascular geometry extracted from in vivo microvascular microscopy. The simulation parameters are $k_{p e}=k_{0}$ and $D_{C A}=D_{0}$. Concentration map $\mathbf{C}$ (a) and magnetic field perturbation $\Delta \mathbf{B}(\mathrm{b})$ are represented at the last simulation time point $\left(t=T_{F}\right)$. (c) Concentration profiles derived from the simulated MR signal using Eqs.[16-17] at 3 different $T_{E}$. The black lines correspond to the fit obtained with the Toft model. Plane size $300 \times 300 \mu m^{2}$. doi:10.1371/journal.pone.0057636.g011 

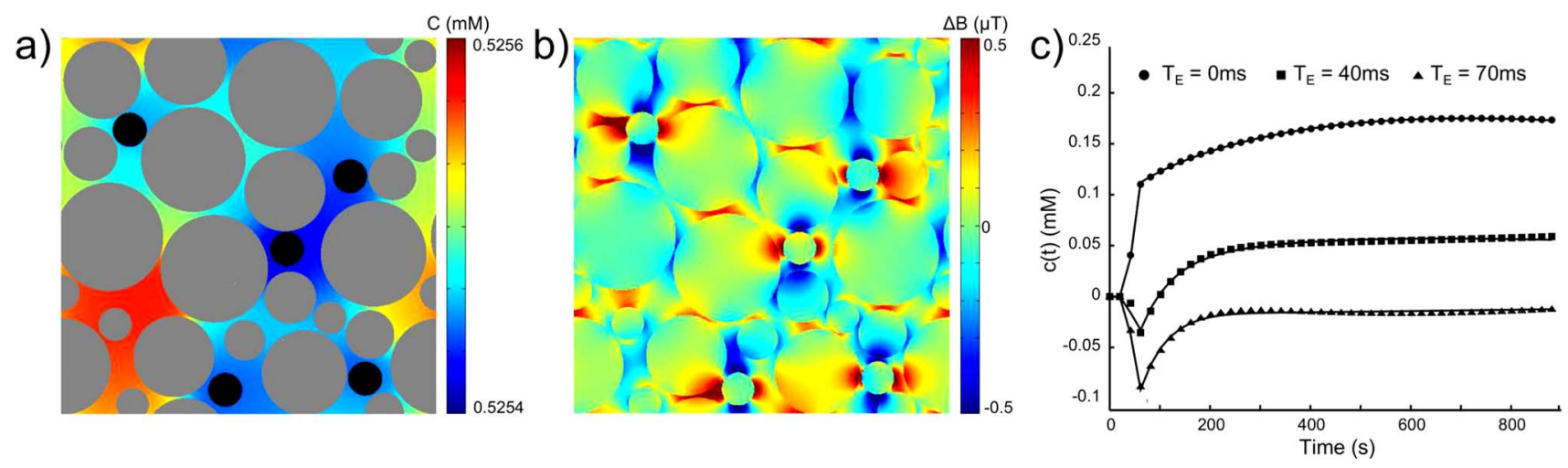

Figure 12. Example of the simulation with impermeable cells placed in the extravascular space. The simulation parameters are: $k_{p e}=k_{0}$ and $D_{C A}=D_{\text {free }}$. At $t=T_{F}$ (a) Concentration map $\mathbf{C}\left(T_{F}\right)$ with vessels in black and cells in grey. (b) Magnetic field perturbation $\Delta \mathbf{B}\left(T_{F}\right)$. (c) Concentration profiles derived from the simulated MR signal and using Eqs.[16-17] at 3 different $T_{E}$. The black lines correspond to the fit obtained with the Toft model. Note the fluctuations in the concentration profiles obtained at long $T_{E}$. This can be ascribed to the additional magnetic field perturbations induced by the cell interfaces which balance the signal enhancement. Plane size $70 \times 70 \mu m^{2}$. doi:10.1371/journal.pone.0057636.g012

adapted for arbitrary vessel geometry, but not for arbitrary susceptibility distribution.

By adapting the Fourier based approach to 2D, we decreased the computation time by about 400 times compared to the analytical approach. In $2 \mathrm{D}$, the single vessel geometry yielded a $T_{2}^{*}$ shorter than what was obtained with multiple vessels. Note that, due to the FFT, the single vessel geometry actually models a periodic vessel distribution. It thus appears that the regularity of the vessel arrangement has an impact on the eventual $T_{2}^{*}$ of the voxel. With a single vessel arrangement, the dipolar effect is spread uniformly over the plane and does not overlap with that of other vessels. Additionally, the distance between any pixel of the plane and a vessel is minimized. Thereby, one maximizes the dipolar effects of the vessel over every points of the lattice. $T_{2}^{*}$ appears sensitive to the vessel arrangements (with constant $B V f$ ), as observed on Fig. 7 where the standard deviation on $T_{2}^{*}$ arises from ten different vessel arrangements. Note that this standard deviation is relatively modest (about 7\%), despite the use of only 5 vessels in each arrangement. Further studies are required to determine the optimal number of vessels per arrangement and the optimal number of arrangements to obtain reliable results in an optimized computation time.

Single or multi-vessel, 2D, geometries yielded shorter $T_{2}^{*}$ than $3 \mathrm{D}$ vascular geometries with comparable BVf (Fig. 6). This difference comes from the fact that the magnetic field perturbation depends on the vessel orientation within $B_{0}$. When all vessels are perpendicular to $B_{0}$, the magnetic field perturbation is maximized and the $T_{2}^{*}$ is reduced compared to what would be obtained with a random distribution of vessel orientations. Interestingly, we observed that averaging the signal from three orthogonal planes yielded $T_{2}^{*}$ comparable to what was obtained with $3 \mathrm{D}$ approaches. One should keep in mind that this pseudo-3D approach requires the use of microvascular characteristics representative of the $3 \mathrm{D}$ distribution ( $B V f$, vessel diameter, vessel arrangements).

\section{Limitations}

The main limitation of the $2 \mathrm{D}$ approach is related to the simulation of the flow within the voxel. Since only a plane is considered in our approach, one can not model plug-flow where CA concentration varies along the capillary bed. The study of the residue function is also harshly limited in our approach. The dispersion of the bolus brought by limited flow and the leakage can be modeled but the dispersion along the vascular tree can not be taken into account easily. With a sufficient number of vessels spread out within the plane, one way to overcome this limitation might be to define different residue functions for each vessel which would model the dispersion of the bolus at different length along the vascular paths. That is to take a section of a $3 \mathrm{D}$ voxel with mixture of arterioles, capillaries and venules as the one used in [45]. Note also that the well-mixedness assumption of the blood compartment may not stand in vivo.

As the expense of computational time, these two restrictions can be overcome by extending our approach to a $3 \mathrm{D}$ voxel. In that case, vessels are usually modeled by straight cylinders. One could also used 3D microvascular networks, such as the one recently studied by Guibert et al [46]. However, the periodic boundary condition does not stand any longer in 3D. One usually deals with this limitation by considering only the MR signal provided by an inner volume where water molecules have not undergoe harsh distortion of the magnetic field caused by discontinuity at the border. However, this can not be used with a reasonable voxel size in the scope of long DCE-MRI simulation where transport of the water and the CA must remain coherent for several minutes. Recently, an elegant solution to this problem has been considered in modeling the vascular network generated by a random walker moving with a significant inertia under periodic boundary conditions [47].

Another strong assumption of our model is related to the free diffusion of water molecules. The permeability of the membranes to water affects the contrast of the MR signal. Different regimes have been considered into the past [7] and means to measure water permeability at the vascular wall are still under development [48]. Based on the approach used for CA transport in this study, the water transport across membranes could be modeled. Thereby, transcytolemnal water exchange could be accounted for [49] at the expense of computational time.

The specific saturation-recovery sequence used in this study may appear very specific. In particular TR is longer that usual. This is due to the specific requirements of DCE-MRI experiments acquired with spirals readout [26]. This however may lead to peak saturation when estimating the AIF in vivo. Other MR sequences can be modeled with our approach such as spoiled GE or inversion-recovery sequences. RF spoiling can easily be implemented. For gradient spoiling, the gradient strength must be 
adapted to the lattice size so that the phase evolution of the water molecules remains coherent at the edges of the lattice. Such a sequence is provided with the source code but additional validation steps are required.

When applying the Tofts model for the analysis, an exact AIF was assumed. The AIF estimation is critical in DCE and is often considered as the main source of error in the permeability estimate. An alternate way is to derive the AIF from the MR signal provided by the blood compartment. This would be more similar to an in vivo situation and the AIF peak saturation effect could be investigated depending on the MR sequence used.

To be more practical the simulation should also incorporate a noise model for the signal. This was not included in the model since the aims was to disentangle the various contributions to the signal. Given the small impact of the CA diffusion on the permeability, it might be worthwhile to confirm if the effects described are observed in presence of noise.

The MR simulation strategies proposed in this study could also be associated to other physiological simulation environments which take into account more complex and realistic compartment models (for instance MMID4, Multiple indicator, Multiple path, Indicator Dilution 4 region model, National Simulation Resource, Department of Bioengineering, University of Washington, Seattle, WA, USA). Extensions of the algorithm to the fields of DSC-MRI or arterial spin labeling could also be considered.

This simulation source code is under the GNU GPL license and is available at http://neurosciences.ujf-grenoble.fr/equipe5.

\section{Conclusion}

We proposed a versatile $2 \mathrm{D}$ simulation tool to model the MR signal in DCE-MRI experiments. We presented how we combined the compartment approach used for DCE-MRI analysis with the

\section{References}

1. Batchelor TT, Sorensen AG, Tomaso Ed, Zhang WT, Duda DG, et al. (2007) AZD2171, a pan-VEGF receptor tyrosine kinase inhibitor, normalizes tumor vasculature and alleviates edema in glioblastoma patients. Cancer Cell 11: 8395.

2. Lemasson B, Christen T, Tizon X, Farion R, Fondraz N, et al. (2011) Assessment of multiparametric MRI in a human glioma model to monitor cytotoxic and anti-angiogenic drug effects. NMR in Biomedicine 24: 473-482.

3. Sourbron SP, Buckley DL (2012) Tracer kinetic modelling in MRI: estimating perfusion and capillary permeability. Physics in Medicine and Biology 57: R1R33.

4. Ostergaard L (2005) Principles of cerebral perfusion imaging by bolus tracking. Journal of Magnetic Resonance Imaging 22: 710-717.

5. Franiel T, Hamm B, Hricak H (2011) Dynamic contrast-enhanced magnetic resonance imaging and pharmacokinetic models in prostate cancer. European Radiology 21: 616-626.

6. Marinovich ML, Sardanelli F, Ciatto S, Mamounas E, Brennan M, et al. (2012) Early prediction of pathologic response to neoadjuvant therapy in breast cancer: Systematic review of the accuracy of MRI. Breast (In Press)

7. Donahue KM, Weisskoff RM, Burstein D (1997) Water diffusion and exchange as they inuence contrast enhancement. Journal of Magnetic Resonance Imaging 7: $102-110$.

8. Tofts PS (1997) Modeling tracer kinetics in dynamic gd-DTPA MR imaging. Journal of Magnetic Resonance Imaging 7: 91-101.

9. Brix G, Kiessling F, Lucht R, Darai S, Wasser K, et al. (2004) Microcirculation and microvasculature in breast tumors: pharmacokinetic analysis of dynamic MR image series. Magnetic Resonance in Medicine 52: 420-429.

10. Buckley DL, Kershaw LE, Stanisz GJ (2008) Cellular-interstitial water exchange and its effect on the determination of contrast agent concentration in vivo: dynamic contrast-enhanced MRI of human internal obturator muscle. Magnetic Resonance in Medicine 60: 1011-1019.

11. Donaldson SB, West CML, Davidson SE, Carrington BM, Hutchison G, et al. (2010) A comparison of tracer kinetic models for T1-weighted dynamic contrastenhanced MRI: application in carcinoma of the cervix. Magnetic Resonance in Medicine 63: 691-700.

12. Yablonskiy DA, Haacke EM (1994) Theory of NMR signal behavior in magnetically inhomogeneous tissues: the static dephasing regime. Magnetic Resonance in Medicine 32: 749-763. physical mechanisms involved in MR contrast. Additionally, we provided a means to efficiently simulate the diffusion of the CA in presence of impermeable compartments. While our results are consistent with in vivo results, some improvements and optimizations are still required. The variability of the MR signal across different sets of vascular networks must be studied (number of vessels and distribution in space). The model of the blood flow can be refined and the effect of the arising magnetic field perturbations due to extravascular cell interfaces and CA leakage requires further investigations.

Many other perfusion studies may be foreseen using the proposed approach. Different MRI sequences can be investigated. Different biophysical models used to analyze DCE experiments can be compared. Various techniques employed to correct for CA extravasation in DSC experiments can be evaluated. The shape of the AIF can also be optimized. These studies may be performed at all magnetic fields and for different tissue types, adjusting parameters such as cell porosity, cell magnetic susceptibility, vessel radius or density. These numerical approaches, in addition to providing a means to deepen our understanding of DCE-MRI, are extremely desirable from an ethical and a financial points of view.

\section{Acknowledgments}

We gratefully acknowledge support from the CNRS/IN2P3 Computing Center (Lyon/Villeurbanne - France) for providing a significant amount of the computing ressources needed for this work.

\section{Author Contributions}

Conceived and designed the experiments: NP CD EB. Performed the experiments: NP CD. Analyzed the data: NP CD. Contributed reagents/ materials/analysis tools: NP CD FM TC. Wrote the paper: NP CD TC EB.

13. Kiselev VG, Posse S (1998) Analytical theory of susceptibility induced NMR signal dephasing in a cerebrovascular network. Physical Review Letters 81: 5696.

14. Jensen JH, Chandra R (2000) Strong field behavior of the NMR signal from magnetically heterogeneous tissues. Magnetic Resonance in Medicine 43: 226236.

15. Sukstanskii AL, Yablonskiy DA (2003) Gaussian approximation in the theory of MR signal formation in the presence of structure-specific magnetic field inhomogeneities. Journal of Magnetic Resonance 163: 236-247.

16. Sourbron S, Heilmann M, Biffar A, Walczak C, Vautier J, et al. (2009) Bolustracking MRI with a simultaneous $\mathrm{T} 1-$ and $\mathrm{T} 2 *$-measurement. Magnetic Resonance in Medicine 62: 672-681.

17. Schmiedeskamp H, Straka M, Newbould RD, Zaharchuk G, Andre JB, et al (2012) Combined spinand gradient-echo perfusion-weighted imaging. Magnetic Resonance in Medicine 68: 30-40.

18. Quarles CC, Gochberg DF, Gore JC, Yankeelov TE (2009) A theoretical framework to model DSCMRI data acquired in the presence of contrast agent extravasation. Phys Med Biol 54: 5749-5766.

19. Sourbron S, Heilmann M, Walczak C, Vautier J, Schad LR, et al. (2012 T2*relaxivity contrast imaging: First results. Magnetic Resonance in Medicine (In Press)

20. Salomir R, de Senneville BD, Moonen CT (2003) A fast calculation method for magnetic field inhomogeneity due to an arbitrary distribution of bulk susceptibility. Concepts in Magnetic Resonance B 19: 26-34.

21. Marques J, Bowtell R (2005) Application of a fourier-based method for rapid calculation of field inhomogeneity due to spatial variation of magnetic susceptibility. Concepts in Magnetic Resonance Part B: Magnetic Resonance Engineering 25B: 65-78.

22. Bandettini PA, Wong EC (1995) Effects of biophysical and physiologic parameters on brain activation-induced $\mathrm{R} 2 *$ and $\mathrm{R} 2$ changes: Simulations using a deterministic diffusion model. International Journal of Imaging Systems and Technology 6: 133-152.

23. Klassen LM, Menon RS (2007) NMR simulation analysis of statistical effects on quantifying cerebrovascular parameters. Biophysical Journal 92: 1014-1021.

24. Valable S, Lemasson B, Farion R, Beaumont M, Segebarth G, et al. (2008) Assessment of blood volume, vessel size, and the expression of angiogenic factors in two rat glioma models: a longitudinal in vivo and ex vivo study. NMR in Biomedicine 21: 1043-1056. 
25. Troprès I, Lamalle L, Péoc'h M, Farion R, Usson Y, et al. (2004) In vivo assessment of tumoral angiogenesis. Magnetic Resonance in Medicine 51: 533 541.

26. Beaumont M, Lemasson B, Farion R, Segebarth C, Rémy C, et al. (2009) Characterization of tumor angiogenesis in rat brain using iron-based vessel size index MRI in combination with gadoliniumbased dynamic contrast-enhanced MRI. Journal of Cerebral Blood Flow Metabolism 29: 1714-1726.

27. Dobre M, Ugurbil K, Marjanska M (2007) Determination of blood longitudinal relaxation time (T1) at high magnetic field strengths. Magnetic Resonance Imaging 25: 733-735.

28. Zhao JM, Clingman CS, Nrvinen MJ, Kauppinen RA, van Zijl PCM (2007) Oxygenation and hematocrit dependence of transverse relaxation rates of blood at 3T. Magnetic Resonance in Medicine 58: 592-597.

29. Kettunen MI, Sierra A, Närväinen MJ, Valonen PK, Yl-Herttuala S, et al. (2007) Low spin-lock field T1 relaxation in the rotating frame as a sensitive MR imaging marker for gene therapy treatment response in rat glioma. Radiology 243: 796-803.

30. Deistung A, Rauscher A, Sedlacik J, Stadler J, Witoszynskyj S, et al. (2008) Susceptibility weighted imaging at ultra high magnetic field strengths: theoretical considerations and experimental results. Magnetic Resonance in Medicine 60: 1155-1168.

31. Christen T, Lemasson B, Pannetier N, Farion R, Segebarth C, et al. (2011) Evaluation of a quantitative blood oxygenation level-dependent (qBOLD) approach to map local blood oxygen saturation. NMR in Biomedicine 24: 393403.

32. Le Bihan D (1995) Di_usion and Perfusion Magnetic Resonance Imaging: Applications to Functional Mri. Raven Press.

33. Marty B, Flament J, Giraudeau C, Le Bihan D, Mériaux S (2010) Apparent diffusion coefficient of gd-based contast agents assessed in vivo in the rat brain using dynamic T1 mapping. In: ISMRM 2010. Stockholm, Sweden.

34. Hall MG, Alexander DC (2009) Convergence and parameter choice for montecarlo simulations of diffusion MRI. IEEE Transactions on Medical Imaging 28: 1354-1364.

35. Christen T, Zaharchuk G, Pannetier N, Serduc R, Joudiou N, et al. (2012) Quantitative MR estimates of blood oxygenation based on T2*: a numerical study of the impact of model assumptions. Magnetic Resonance in Medicine 67: 1458-1468.

36. Kennan RP, Zhong J, Gore JC (1994) Intravascular susceptibility contrast mechanisms in tissues. Magnetic Resonance in Medicine 31: 9-21.
37. Boxerman JL, Hamberg LM, Rosen BR, Weisskoff RM (1995) MR contrast due to intravascular magnetic susceptibility perturbations. Magnetic Resonance in Medicine 34: 555-566.

38. Pathak AP, Ward BD, Schmainda KM (2008) A novel technique for modeling susceptibility-based contrast mechanisms for arbitrary microvascular geometries: the finite perturber method. Neuroimage 40: 1130-1143.

39. Pellerin M, Yankeelov TE, Lepage M (2007) Incorporating contrast agent diffusion into the analysis of DCE-MRI data. Magnetic Resonance in Medicine 58: 1124-1134.

40. Pollack GH (2003) The role of aqueous interfaces in the cell. Adv Colloid Interface Sci 103: 173-196.

41. Le Bihan D (2007) The 'wet mind': water and functional neuroimaging. Phys Med Biol 52: R57-R90.

42. Kiselev VG (2001) On the theoretical basis of perfusion measurements by dynamic susceptibility contrast MRI. Magnetic Resonance in Medicine 46: 1113-1122.

43. Dickson JD, Ash TWJ, Williams GB, Harding SG, Carpenter TA, et al. (2010) Quantitative BOLD: the effect of diffusion. Journal of Magnetic Resonance Imaging 32: 953-961.

44. Risser L, Plouraboué F, Steyer A, Cloetens P, Le Duc G, et al. (2007) From homogeneous to fractal normal and tumorous microvascular networks in the brain. Journal of Cerebral Blood Flow and Metabolism 27: 293-303.

45. Xu C, Schmidt WU, Galinovic I, Villringer K, Hotter B, et al. (2012) The potential of microvessel density in prediction of infarct growth: A two-month experimental study in vessel size imaging. Cerebrovascular Diseases 33: 303309.

46. Guibert R, Fonta C, Risser L, Plouraboué F (2012) Coupling and robustness of intra-cortical vascular territories. NeuroImage 62: 408-417.

47. Novikov DS, Reisert M, Kiselev VG (2012) Susceptibilty-induced increase of aparent diffusion coefficient: BOLD effect behind diffusion fMRI. In: ISMRM 2012. Melbourne, Australia.

48. St Lawrence KS, Owen D, Wang DJJ (2012) A two-stage approach for measuring vascular water exchange and arterial transit time by diffusionweighted perfusion MRI. Magnetic Resonance in Medicine 67: 1275-1284.

49. Yankeelov TE, Luci JJ, DeBusk LM, Lin PC, Gore JC (2008) Incorporating the effects of transcytolemmal water exchange in a reference region model for DCEMRI analysis: theory, simulations, and experimental results. Magnetic Resonance in Medicine 59: 326-335. 